\title{
Effects of $C P T$-odd terms of dimensions three and five on electromagnetic propagation in continuous matter
}

\author{
Pedro D. S. Silva $\odot,{ }^{*}$ Letícia Lisboa-Santos $\odot,{ }^{\dagger}$ Manoel M. Ferreira, Jr. $\odot{ }^{\dagger}{ }^{\dagger}$ and Marco Schreck $\odot^{\S}$ \\ Departamento de Física, Universidade Federal do Maranhão, Campus Universitário do Bacanga, \\ São Luís (MA), 65080-805, Brazil
}

(Received 10 September 2021; revised 10 October 2021; accepted 3 November 2021; published 27 December 2021)

\begin{abstract}
In this work we study how CPT-odd Maxwell-Carroll-Field-Jackiw (MCFJ) electrodynamics as well as a dimension-5 extension of it affect the optical activity of continuous media. The starting point is dimension-3 MCFJ electrodynamics in matter whose modified Maxwell equations, permittivity tensor, and dispersion relations are recapitulated. Corresponding refractive indices are achieved in terms of the frequency and the vector-valued background field. For a purely timelike background, the refractive indices are real. Their associated propagation modes are circularly polarized and exhibit birefringence. For a purely spacelike background, one refractive index is always real and the other can be complex. The circularly polarized propagating modes may exhibit birefringence and dichroism (associated with absorption). Subsequently, we examine a dimension-five MCFJ-type electrodynamics, previously scrutinized in the literature, in a continuous medium. Following the same procedure, we find the refractive indices from a sixth-order dispersion equation. For a purely timelike background, three distinct refractive indices are obtained, one of them being real and two being complex. They are associated with two circularly polarized propagating modes that exhibit birefringence or dichroism, depending on the frequency range. Scenarios of propagation and absorption analogous to those found in dispersive dielectrics are also observed for purely spacelike background configurations. We conclude by comparing the dimension-three and five results and by emphasizing the richer phenomenology of the propagating modes in the higher-derivative model. Our results are applicable in the realm of Weyl semimetals.
\end{abstract}

DOI: $10.1103 /$ PhysRevD.104.116023

\section{INTRODUCTION}

The dynamics of electromagnetic fields in continuous media is governed by the Maxwell equations, supplemented by constitutive relations [1,2] that describe the response of the medium to external, applied electromagnetic fields. In vacuo these relations simply read $\mathbf{D}=\epsilon_{0} \mathbf{E}$ and $\mathbf{H}=\mu_{0}^{-1} \mathbf{B}$, where $\epsilon_{0}$ and $\mu_{0}$ are the electric vacuum permittivity and magnetic permeability constant, respectively. The first constitutive relation takes into account the electric polarization in a dielectric medium, while the latter includes magnetization effects. For an isotropic medium, the constitutive relations are $\mathbf{D}=\epsilon \mathbf{E}$ and $\mathbf{H}=\mu^{-1} \mathbf{B}$ with scalar material parameters $\epsilon, \mu$ replacing $\epsilon_{0}, \mu_{0}$ governing

\footnotetext{
*pedro.dss@discente.ufma.br

†leticia.lisboa@discente.ufma.br

*manoel.messias@ufma.br, manojr.ufma@gmail.com

§marco.schreck@ufma.br
}

Published by the American Physical Society under the terms of the Creative Commons Attribution 4.0 International license. Further distribution of this work must maintain attribution to the author(s) and the published article's title, journal citation, and DOI. Funded by SCOAP ${ }^{3}$. the vacuum properties. More involved constitutive relations appear in two main scenarios: (i) anisotropic media, where electric permittivity and magnetic permeability become tensors (cf. uniaxial and biaxial crystals [3-8], Weyl semimetals $[9,10]$, and magnetized materials $[11,12])$; (ii) novel effects in matter described by extended constitutive relations that introduce additional electric and magnetic responses, encoded as linear functions of the type $\mathbf{D}=\mathbf{D}(\mathbf{E}, \mathbf{B})$ and $\mathbf{H}=\mathbf{H}(\mathbf{E}, \mathbf{B})$, in general. This happens, for instance, in bi-isotropic media [13-16], chiral materials [17], topological insulators [18-23], relativistic electron gases [24], axion electrodynamics [25-27], and Lorentz-violating electrodynamics [28,29], as well.

Generalizations of electrodynamics including higherderivative terms have also been conceived in the literature. First studies of electrodynamics in the presence of higher derivatives are ascribed to Bopp [30] in 1940, and to Podolsky [31,32] in 1942. Their model implements a second-order derivative term $\theta^{2} \partial_{\alpha} F^{\alpha \beta} \partial_{\sigma} F_{\beta}^{\sigma}$ into the Maxwell Lagrangian in vacuo. The modified MaxwellPodolsky equations, sometimes called Bopp-Podolsky equations, yield a photon mass term, proportional to the inverse of the Podolsky parameter $\theta$. Furthermore, this extension exhibits two dispersion relations, the usual one 
from Maxwell theory and a second one ascribed to a massive mode.

The constraint structure of this theory was investigated in [33] and its quantization was performed in [34]. Further aspects of the Maxwell-Podolsky model were examined, including the problems of self-force and self-interaction [35,36], Green functions and classical solutions [37], multipole expansion for fields in the static regime [38], symmetrization/conservation of the energy-momentum tensor [39], its consistency based on the BRST approach [40], quantum field theoretic properties $[41,42]$ as well as other characteristics [43].

A further example for a generalization of Maxwell electrodynamics is provided by Lee-Wick electrodynamics 44,45]], which introduces the modification $F^{\mu \nu} \square F_{\mu \nu}$ [46-48]. The higher-derivative Lee-Wick term can arise as a quantum correction in models with a nonminimal coupling between the gauge and fermionic fields [49].

In the past years, higher-derivative contributions have also been examined in the context of Lorentz-violating theories. The possibility of Lorentz invariance violation (LV) was proposed in the context of physics at the Planck scale such as strings [50]. Presently, the Standard-Model Extension (SME) [51], incorporating fixed background tensor fields coupled to the dynamical Standard-Model fields, is usually employed as the main framework to parametrize it. Lorentz violation in the electromagnetic sector of the SME occurs by means of a $C P T$-odd or a $C P T$-even term [52-55]. The $C P T$-odd part is represented by the Carroll-Field-Jackiw (CFJ) contribution [56-62], which has found applications in condensed-matter systems that violate parity $(P)$ and time reversal $(T)$ symmetry [63] as well as those endowed with the chiral magnetic effect [64-67] and the anomalous Hall effect [68].

Nonminimal extensions of the SME were proposed including higher-derivative terms with mass dimensions greater than four (in natural units) [69-71]. In this context, the Myers-Pospelov model [72,73] was a pioneering proposal focusing on dimension-five contributions. Recently, classical aspects of a modified, higher-derivative electrodynamics in vacuo were discussed in [74,75], including the derivation of the gauge propagator, the dispersion relations as well as an analysis of causality, unitary, and stability of the modes. Profound analyses were accomplished for the Maxwell-Podolsky electrodynamics modified by $C P T$ even, dimension-six terms [74] and for a CPT-odd, dimension-five electrodynamics [75]. Some results of Ref. [75] were revisited and discussed in Ref. [76]. Nonminimal higher-derivative models have also been used to study the interaction energy between electromagnetic sources [77] and the thermodynamic properties of electrodynamic systems [78] as well as in the context of Horăva-Lifshitz electrodynamics [79] and radiative corrections [80].

The plethora of nonminimal LV theories on the one hand and the optical properties of new materials $[13,81]$ on the other hand is a strong motivation for investigating higherderivative effects on the propagation of electromagnetic waves in dielectric substrates, including aspects of optical activity and dichroism. In this sense, the present work is devoted to analyzing the behavior of a continuous medium governed by a MCFJ-type electrodynamics in the absence and presence of higher-derivative terms.

This paper is outlined as follows. In Sec. II, we briefly review the covariant description of electrodynamics in macroscopic materials recapitulating the definition of birefringence (double refraction) and dichroism. We will be considering simple matter as opposed to designed materials with highly peculiar properties such as metamaterials [82-84]. In Sec. III, we present aspects of the MCFJ electrodynamics in a ponderable medium, showing that the timelike CFJ background yields birefringence, while the spacelike one provides birefringence and dichroism. In Sec. IV, we discuss the higher-derivative MCFJ electrodynamics in continuous matter based on more involved scenarios. Finally, we present our main findings in Sec. V. Throughout the paper, we employ natural units with $\hbar=c=1$ unless otherwise stated. Furthermore, our signature choice for the Minkowski metric $\eta_{\mu \nu}$ is $(+,-,-,-)$.

\section{ELECTRODYNAMICS IN SIMPLE MATTER}

In a continuous medium, the electromagnetic properties are described by the Maxwell equations [1,2] combined with the constitutive relations. For a general linear, homogeneous, and anisotropic medium, the constitutive relations can be written as

$$
\begin{aligned}
& \mathbf{D}=\epsilon \cdot \mathbf{E}+\gamma \cdot \mathbf{B}, \\
& \mathbf{H}=\tilde{\gamma} \cdot \mathbf{E}+\mu^{-1} \cdot \mathbf{B},
\end{aligned}
$$

where $\epsilon$ and $\mu$ represent the electric permittivity and magnetic permeability tensors [8,13-16], respectively. The tensor $\gamma$ measures the magnetic contribution to the electric displacement field $\mathbf{D}$, while $\tilde{\gamma}$ represents the electric contribution to the magnetic field $\mathbf{H}$. Regarding the structure of constitutive relations (1a) and (1b), interesting scenarios of electromagnetic behavior may occur, e.g., in anisotropic media $[4,5,7,8,13-16]$, Weyl semimetals $[9,10,85]$, magnetized ferrites $[11,12]$, and in chiral media and topological insulators [19-21]. Besides Eq. (1), general constitutive relations for the current density, $\mathbf{J}=\mathbf{J}(\mathbf{E}, \mathbf{B})$, can also be considered. As an example, a dielectric system endowed with a magnetic conductivity has recently been examined at the classical level [86], reporting interesting effects such as an induced electric conductivity, isotropic birefringence, and parity violation. A physical realization of the antisymmetric magnetic current examined in [86] via a microscopic model was addressed in Ref. [87]. 
The constitutive relations in Eq. (1) can be naturally encoded in the Lagrange density formalism via

$$
\mathcal{L}=-\frac{1}{4} G^{\mu \nu} F_{\mu \nu}-A_{\mu} J^{\mu},
$$

with the four-potential $A_{\mu}$, the electromagnetic field strength tensor $F_{\mu \nu}=\partial_{\mu} A_{\nu}-\partial_{\nu} A_{\mu}$, and an external, conserved four-current $J^{\mu}$. Furthermore, the antisymmetric tensor $G^{\mu \nu}$ is defined as [8]

$$
G^{\mu \nu} \equiv \frac{1}{2} \chi^{\mu \nu \alpha \beta} F_{\alpha \beta},
$$

with $\chi^{\mu \nu \alpha \beta}$ being the constitutive tensor that parametrizes the medium's response to the applied electromagnetic fields [88]. The constitutive tensor satisfies the following symmetry properties:

$$
\begin{aligned}
& \chi^{\mu \nu \alpha \beta}=-\chi^{\nu \mu \alpha \beta}, \\
& \chi^{\mu \nu \alpha \beta}=-\chi^{\mu \nu \beta \alpha}, \\
& \chi^{\mu \nu \alpha \beta}=\chi^{\alpha \beta \mu \nu},
\end{aligned}
$$

compatible with the symmetries of the field strength tensor. The Euler-Lagrange equation applied to the Lagrangian of Eq. (2a) (a complete derivation is presented in Appendix A) yields

$$
\partial_{\mu} G^{\mu \nu}=J^{\nu} .
$$

The homogenous Maxwell equations are obtained from the Bianchi identity valid for the curvature $F_{\mu \nu}$ of the principal $U(1)$ fiber bundle:

$$
\partial_{\mu} \tilde{F}^{\mu \nu}=0, \quad \tilde{F}^{\mu \nu}=\frac{1}{2} \epsilon^{\mu \nu \alpha \beta} F_{\alpha \beta},
$$

which is why the latter are not affected by the presence of the medium. A straightforward calculation from Eqs. (4), (5) leads to the well-known Maxwell equations in simple matter, namely:

$$
\begin{array}{r}
\nabla \cdot \mathbf{D}=\rho, \\
\nabla \times \mathbf{H}-\partial_{t} \mathbf{D}=\mathbf{J}, \\
\nabla \cdot \mathbf{B}=0, \\
\nabla \times \mathbf{E}+\partial_{t} \mathbf{B}=0,
\end{array}
$$

where the constitutive relations for the electric displacement field $\mathbf{D}$ and magnetic field $\mathbf{H}$ are given in Eq. (1). The Maxwell equations of Eq. (6) and the constitutive relations given by Eq. (1) allow us to describe the dynamics of electromagnetic fields in simple matter.

In crystals and generic anisotropic media, the dispersion relations are given by the Fresnel equation [2]. The latter is obtained from algebraic manipulations of the Maxwell equations for continuous media whose properties are encoded in the permittivity tensor. In such a medium, the dielectric permittivity tensor is a function of the frequency $\omega$, the wave vector $\mathbf{k}$, and the (external) magnetic field $\mathbf{B}$ (or the magnetization, alternatively), $\epsilon_{i j}=\epsilon_{i j}(\omega, \mathbf{k}, \mathbf{B})$, and can be expanded as [81]

$\epsilon_{i j}(\omega, \mathbf{k}, \mathbf{B})=\epsilon_{i j}^{0}+\alpha_{i j l} k_{l}+\beta_{i j l} B_{l}+\gamma_{i j a b} k_{a} B_{b}+\ldots$

The first term, $\epsilon_{i j}^{0}$, is the usual permittivity tensor of a dielectric. The second term, $\alpha_{i j l} k_{l}$, is a signature of spatial inversion symmetry breaking. It implies an optical activity that becomes manifest via linear birefringence or a rotation of the oscillation plane of linearly polarized light $[4,5]$. The third term, $\beta_{i j l} B_{l}$, is associated with a fixed external magnetic field or magnetization and leads to a violation of time reversal symmetry. It gives rise to a magneto-optical activity via the Faraday or the Cotton-Mouton effect [81].

As already mentioned, a known consequence of the optical activity of a medium is linear birefringence, occurring when two circularly polarized modes of opposite chiralities, with refractive indices $n_{+}$and $n_{-}$, respectively, have different phase velocities, $c / n_{+}$and $c / n_{-}$. This property implies a rotation of the polarization plane of a linearly polarized wave. The latter phenomenon is quantified by the specific rotatory power $\delta$, which measures the rotation of the oscillation plane of linearly polarized light per unit traversed length in the medium. It is defined as

$$
\delta=-\frac{\omega}{2}\left[\operatorname{Re}\left(n_{+}\right)-\operatorname{Re}\left(n_{-}\right)\right],
$$

where $n_{+}$and $n_{-}$are associated with left and right-handed circularly polarized waves, respectively.

Another interesting effect occurring in anisotropic crystals, dichroism, takes place when one polarization component is more strongly absorbed than the other. Obviously, this property is linked to the imaginary part of the refractive index. The difference of absorption of left and right-handed circularly polarized modes [81] is given by the dichroism coefficient [see Appendix B for the derivation of Eqs. (8), (9)]:

$$
\delta_{\mathrm{d}}=-\frac{\omega}{2}\left[\operatorname{Im}\left(n_{+}\right)-\operatorname{Im}\left(n_{-}\right)\right] .
$$

In the following, we examine two models of modified electrodynamics in continuous matter: the first one governed by the MCFJ Lagrangian and the second one by a higher-derivative MCFJ-type Lagrangian. 


\section{MCFJ MODEL IN A CONTINUOUS MEDIUM}

In principle, MCFJ electrodynamics has connections with systems of chiral fermions, in particular, the chiral magnetic effect (CME), the anomalous Hall effect (AHE), and the anomalous generation of charge, besides birefringence effects. In Ref. [63], the Maxwell equations in vacuo modified by the CFJ background were obtained, which yields the terms ascribed to the CME and AHE. In this section, we examine aspects of the MCFJ electrodynamics embedded in a continuous medium. The latter plays a role for condensed-matter systems such as Weyl semimetals [89]. These novel materials are characterized by an even number of Weyl cones separated from each other in momentum space. In the vicinity of these cones, electrons behave as massless particles and have a certain Fermi velocity associated with them, whereupon their description via the Weyl equation is admissible. Having a microscopic realization of such a material at hand, it can be consistently described in the context of effective field theory via a $b$ term of the minimal SME fermion sector [51]. The modified Dirac theory is frequently recast into the form $[85,90]$

$$
\mathcal{L}=\bar{\psi}\left[\gamma^{\mu}\left(\mathrm{i} \partial_{\mu}-b_{\mu} \gamma^{5}\right)-m\right] \psi,
$$

where $\psi$ is a Dirac spinor field, $\bar{\psi}$ its Dirac conjugate, $m$ the electron mass, $\gamma^{\mu}$ the standard Dirac matrices, and $\gamma^{5}$ is the chiral Dirac matrix. The vector-valued background field $b_{\mu}$ is known to catch the essential properties of a certain class of these materials. Integrating out the fermion fields implies an action for the electromagnetic fields. The latter decomposes into a $C P T$-even part, which gives rise to a nontrivial permittivity and permeability of the system, as well as a $C P T$-odd part corresponding to a CFJ term. We will come back to this point below.

The MCFJ Lagrange density in matter has the form

$$
\mathcal{L}=-\frac{1}{4} G^{\mu \nu} F_{\mu \nu}-\frac{1}{4} \epsilon^{\beta \lambda \mu \nu} V_{\beta} A_{\lambda} F_{\mu \nu}-A_{\mu} J^{\mu},
$$

with the intrinsic vector-valued field $V^{\mu}$. Furthermore, $\epsilon^{\beta \lambda \mu \nu}$ is the Levi-Civita symbol in Minkowski spacetime fulfilling $\epsilon^{\beta \lambda \mu \nu}=-\epsilon_{\beta \lambda \mu \nu}$ and $\epsilon_{0123}=1$. The latter Lagrange density yields the following modified inhomogeneous Maxwell equations:

$$
\begin{array}{r}
\nabla \cdot \mathbf{D}-\mathbf{V} \cdot \mathbf{B}=\rho, \\
\nabla \times \mathbf{H}-\partial_{t} \mathbf{D}-V_{0} \mathbf{B}+\mathbf{V} \times \mathbf{E}=\mathbf{J},
\end{array}
$$

where $\mathbf{V}$ is the spatial part of $V^{\mu}$ and the fields $\mathbf{D}, \mathbf{H}$ fulfill the linear constitutive relations of Eq. (1). Notice that the presence of the tensor $G^{\mu \nu}$ renders the Lagrangian (11) different from the one of Ref. [63], meaning that the modified Maxwell equations of Eq. (12) apply to a
TABLE I. Behavior of the $\mathrm{LV}$ terms of the Lagrangian given by Eq. (13) under $C$ (charge conjugation), $P$ (parity), and $T$ (time reversal).

\begin{tabular}{cccccccc}
\hline \hline & $\mathbf{E}$ & $\mathbf{B}$ & $A_{0}$ & $\mathbf{A}$ & $V_{0}(\mathbf{A} \cdot \mathbf{B})$ & $A_{0}(\mathbf{V} \cdot \mathbf{B})$ & $\mathbf{V} \cdot(\mathbf{A} \times \mathbf{E})$ \\
\hline$C$ & - & - & - & - & + & + & + \\
$P$ & - & + & + & - & - & + & + \\
$T$ & + & - & + & - & + & - & - \\
\hline \hline
\end{tabular}

ponderable medium. Concerning the discrete symmetries, $C$ (charge conjugation), $P$, and $T$, it is worthwhile to recall that the CFJ term is $C P T$-odd and the free part of the Lagrangian in Eq. (11) can be written as:

$$
\begin{aligned}
\mathcal{L} \supset & \frac{1}{2}(\mathbf{E} \cdot \mathbf{D}-\mathbf{B} \cdot \mathbf{H}) \\
& +\frac{1}{2}\left[V^{0}(\mathbf{A} \cdot \mathbf{B})-A^{0}(\mathbf{V} \cdot \mathbf{B})+\mathbf{V} \cdot(\mathbf{A} \times \mathbf{E})\right] .
\end{aligned}
$$

In this sense, the pieces involving $V_{0}$ are $P$-odd and $T$-even, while the terms composed of $\mathbf{V}$ are $P$-even and $T$-odd, as properly shown in Table I. Thus, these terms can induce an optical activity of the medium (in the form of birefringence or dichroism).

Another interesting aspect of MCFJ electrodynamics is that the term $V_{0}$ B plays a significant role in a chiral magnetic current,

$$
\mathbf{J}_{\mathrm{CME}}=\frac{e^{2}}{4 \pi^{2}}(\Delta \mu) \mathbf{B} \equiv \Sigma \mathbf{B},
$$

usually generated in chiral fermion systems $[64-67,86]$. Here, $\Delta \mu \equiv \mu_{R}-\mu_{L}$ is also known as the chiral chemical potential and fermions of electric charge $\pm e$ are considered. In Eq. (14), $\Sigma$ represents an isotropic chiral magnetic conductivity and plays a role equivalent to that of the timelike component $V_{0}$ in Eq. (12b), as pointed out in Ref. [63]. For the present analysis, we consider the usual constitutive relations. We set $\gamma_{i j}=\tilde{\gamma}_{i j}=0$ in Eq. (1), implying

$$
D^{i}=\epsilon_{i j} E^{j}, \quad H^{i}=\left(\mu^{-1}\right)_{i j} B^{j},
$$

which can be restricted to the special scenario of an isotropic medium by choosing configurations proportional to the identity,

$$
\epsilon_{i j}=\epsilon \delta_{i j}, \quad\left(\mu^{-1}\right)_{i j}=\mu^{-1} \delta_{i j},
$$

where $\epsilon$ and $\mu$ are the electric permittivity and magnetic permeability constants, respectively. This approach is equivalent to taking the constitutive relations $\mathbf{D}=\epsilon \mathbf{E}$, and $\mathbf{H}=\mu^{-1} \mathbf{B}$.

In what follows, we implement the latter relations as well as $\mathbf{J}=\sigma \mathbf{E}$, where $\sigma$ is the Ohmic conductivity. 
Furthermore, we employ a plane-wave ansatz for the fields, $\mathbf{E}=\mathbf{E}_{0} e^{\mathrm{i}(\mathbf{k} \cdot \mathbf{r}-\omega t)}, \mathbf{B}=\mathbf{B}_{0} e^{\mathrm{i}(\mathbf{k} \cdot \mathbf{r}-\omega t)}$, and similarly for $\mathbf{D}$ and $\mathbf{H}$ in Eq. (12b). One then gets

$$
\mathrm{i} \mathbf{k} \times \mathbf{B}+\mathrm{i} \mu \epsilon \omega \mathbf{E}-\mu V_{0} \mathbf{B}+\mu \mathbf{V} \times \mathbf{E}-\mu \sigma \mathbf{E}=0,
$$

which can be simplified by using Faraday's law, yielding

$$
\left[\mathbf{k}^{2} \delta_{i j}-k_{i} k_{j}-\omega^{2} \mu \bar{\epsilon}_{i j}\right] E^{j}=0,
$$

where we have defined

$$
\bar{\epsilon}_{i j} \equiv\left(\epsilon+\mathrm{i} \frac{\sigma}{\omega}\right) \delta_{i j}-\frac{\mathrm{i}}{\omega^{2}} \epsilon_{i a j}\left(k_{a} V_{0}-\omega V_{a}\right),
$$

as an effective electric permittivity tensor. Here, $\epsilon_{i j k}$ is the Levi-Civita symbol in three dimensions. The second term on the right-hand side of Eq. (18b), $\epsilon_{i a j} k_{a} V_{0}$, is analogous to the tensor $\alpha_{i j l} k_{l}$ of Eq. (7), which breaks parity invariance. The third term, $\epsilon_{i a j} \omega V_{a}$, breaks time reversal invariance. Both are responsible for the optical activity of the medium, becoming manifest in birefringence, as we shall see.

For a continuous medium, we write $\mathbf{k}=\omega \mathbf{n}$, where $\mathbf{n}$ is a vector pointing along the direction of the wave vector and yielding the refractive index $n=+\sqrt{\mathbf{n}^{2}}$. To permit complex refractive indices, we take $+\sqrt{\mathbf{n}^{2}}$ instead of $|\mathbf{n}|$. The plus sign indicates, in principle, that we discard refractive indices with negative real parts, whenever such could occur. Composites with negative real parts of their refractive indices are called metamaterials [82-84] and such possibilities will not be taken into account. Then, Eq. (18a) becomes

$$
M_{i j} E^{j}=0,
$$

with the tensor

$$
M_{i j}=n^{2} \delta_{i j}-n_{i} n_{j}-\mu \bar{\epsilon}_{i j},
$$

where

$$
\bar{\epsilon}_{i j}=\left(\epsilon+\mathrm{i} \frac{\sigma}{\omega}\right) \delta_{i j}-\frac{\mathrm{i}}{\omega} \epsilon_{i a j}\left(n_{a} V_{0}-V_{a}\right) .
$$

The nontrivial solutions for the electric field are obtained by requiring that the determinant of the matrix $M_{i j}$ vanish, $\operatorname{det}\left[M_{i j}\right]=0$, which yields the dispersion relations that describe wave propagation in the medium. The matrix $M_{i j}$ is explicitly given by

$$
\left[M_{i j}\right]=\mathcal{M}+\mathrm{i} \frac{\mu}{\omega} \mathcal{V}
$$

$\mathcal{M}=\left[n^{2}-\mu\left(\epsilon+\mathrm{i} \frac{\sigma}{\omega}\right)\right] \mathbb{1}_{3}-\left(\begin{array}{ccc}n_{1}^{2} & n_{1} n_{2} & n_{1} n_{3} \\ n_{1} n_{2} & n_{2}^{2} & n_{2} n_{3} \\ n_{1} n_{3} & n_{2} n_{3} & n_{3}^{2}\end{array}\right)$,

$$
\mathcal{V}=\left(\begin{array}{ccc}
0 & V_{3}-V_{0} n_{3} & V_{0} n_{2}-V_{2} \\
V_{0} n_{3}-V_{3} & 0 & V_{1}-V_{0} n_{1} \\
V_{2}-V_{0} n_{2} & V_{0} n_{1}-V_{1} & 0
\end{array}\right),
$$

with the $(3 \times 3)$ identity matrix $\mathbb{1}_{3}$. The dispersion equation follows from $\operatorname{det}\left[M_{i j}\right]=0$ :

$$
\begin{aligned}
0= & \tilde{\epsilon}\left(n^{2}-\mu \tilde{\epsilon}\right)^{2}-\frac{\mu}{\omega^{2}}\left\{\mu \tilde{\epsilon}\left[V_{0}^{2} n^{2}+\mathbf{V}^{2}-2 V_{0}(\mathbf{n} \cdot \mathbf{V})\right]\right. \\
& \left.-\mathbf{V}^{2} n^{2}+(\mathbf{n} \cdot \mathbf{V})^{2}\right\},
\end{aligned}
$$

where

$$
\tilde{\epsilon}=\epsilon+\mathrm{i} \frac{\sigma}{\omega} .
$$

We note that via the choices

$$
\bar{p}^{\mu} \equiv\left(\sqrt{\tilde{\epsilon}} \omega, \frac{\mathbf{k}}{\sqrt{\mu}}\right), \quad \bar{V}^{\mu} \equiv\left(\sqrt{\mu} V^{0}, \frac{\mathbf{V}}{\sqrt{\tilde{\epsilon}}}\right)
$$

our Eq. (21) is equivalent to

$$
\bar{p}^{4}+\bar{p}^{2} \bar{V}^{2}-(\bar{p} \cdot \bar{V})^{2}=0 .
$$

Alternatively, we can introduce an effective metric of the form

$$
\tilde{\eta}_{\mu \nu} \equiv \operatorname{diag}\left(\tilde{\epsilon},-\frac{1}{\mu},-\frac{1}{\mu},-\frac{1}{\mu}\right),
$$

and write the dispersion equation as

$$
\begin{aligned}
0= & (p \cdot \tilde{\eta} \cdot p)^{2} \\
& +\frac{\mu}{\tilde{\epsilon}}\left[(p \cdot \tilde{\eta} \cdot p)(V \cdot \tilde{\eta} \cdot V)-(p \cdot \tilde{\eta} \cdot V)^{2}\right] .
\end{aligned}
$$

In vacuo, where $\epsilon=\mu=1$ and $\sigma=0$, the conventional four-momentum $p^{\mu}=(\omega, \mathbf{k})$ and the preferred spacetime direction $V^{\mu}=\left(V^{0}, \mathbf{V}\right)$ satisfy

$$
p^{4}+p^{2} V^{2}-(p \cdot V)^{2}=0,
$$

as expected. Equation (26) is the well-known dispersion equation of the MCFJ model in vacuo [56,57]. Therefore, we interpret Eq. (23) as the dispersion equation for a generalized MCFJ theory in media. The four-vector $\bar{p}^{\mu}$ of Eq. (22) plays the role of an effective four-momentum that formally satisfies an analogous dispersion equation as 
in vасио when the preferred direction is replaced by $\bar{V}^{\mu}$. The possibility of expressing the dispersion equation in terms of the effective metric in Eq. (24) and the conventional four-momentum $p^{\mu}$ is a different way of understanding this result. The presence of a medium described by the material parameters $\epsilon, \mu$, and $\sigma$ leads to electromagnetic waves obeying an analogous dispersion equation as in vacuum, but with the Minkowski metric replaced by an effective metric.

Now, let us have another look at MCFJ theory in the context of Weyl semimetals. As described in [85], integrating out the fermion fields of the effective field theory stated in Eq. (10) implies a modified electrodynamics described by the first two terms in Eq. (11). Considering the realization of a Weyl semimetal studied in the latter reference leads to a particular choice of $V^{\mu}$ with $V^{0}=0$ and $\mathbf{V}$ pointing along the third spatial axis [compare the modified inhomogeneous Maxwell equations of their Eqs. (31), (34) to our Eqs. (12a), (12b)]. Although the $\mathrm{CFJ}$ term incorporates the most intriguing properties of such materials, we must also take into account that a Weyl semimetal (like any material) is characterized by a nontrivial permittivity (whereas the permeability is often simply to set 1). Thus, the optical response of such a material is very well described by a dispersion equation of the form of Eq. (25) (cf. Eq. (36) in [85]) being a formidable motivation for considering theories such as Eq. (11). The author of the latter paper emphasizes that the presence of the CFJ term implies birefringence in Weyl semimetals.

In order to further understand some properties of MCFJ electrodynamics in a continuous dielectric medium with magnetic properties, we address two main scenarios: (i) a timelike and (ii) a spacelike background field $V^{\mu}$. We choose a non-Ohmic medium as a substrate, which implies $\sigma \mapsto 0$ in Eq. (21).

\section{A. Purely timelike case}

For the purely timelike scenario, $V^{\mu}=\left(V^{0}, \boldsymbol{0}\right)$, Eq. (21) reduces to

$$
\left(n^{2}-\mu \epsilon\right)^{2}-\frac{\mu^{2} V_{0}^{2}}{\omega^{2}} n^{2}=0,
$$

which yields two distinct refractive indices:

$$
n_{ \pm}^{2}=\mu \epsilon+\frac{\mu^{2} V_{0}^{2}}{2 \omega^{2}} \pm \frac{\mu V_{0}}{\omega} \sqrt{\mu \epsilon+\frac{\mu^{2} V_{0}^{2}}{4 \omega^{2}}},
$$

or equivalently

$$
n_{ \pm}=\sqrt{\mu \epsilon+\frac{\mu^{2} V_{0}^{2}}{4 \omega^{2}}} \pm \frac{\mu V_{0}}{2 \omega} .
$$

The latter result is in accordance with the refractive index given in Eq. (25) of Ref. [86] for the diagonal isotropic magnetic conductivity tensor. This is an expected correspondence, since one knows that $V_{0}$ plays the role of a "magnetic conductivity," as remarked below Eq. (14). Note that $n_{ \pm}$are real and positive, allowing both modes to propagate for any frequency, so that an absorbing behavior is not observed here. Furthermore, in the limit of high frequencies, Eq. (28b) provides $n_{ \pm} \mapsto \sqrt{\mu \epsilon}$, recovering the refractive index of a medium with electric permittivity $\epsilon$ and magnetic permeability $\mu$, as described in the context of Maxwell electrodynamics. This behavior is illustrated in Fig. 1, which depicts the refractive indices (28b) in terms of the dimensionless parameter $\omega / V_{0}$ for some values of $\mu$ and $\epsilon$. The mode associated with $n_{+}$exhibits anomalous dispersion, meaning that $\mathrm{d} n_{+} / \mathrm{d} \omega<0$, while $n_{-}$is characterized by normal dispersion. In order to examine the polarization state of the propagation modes, we first rewrite Eq. (27),

$$
n^{2}-\mu \epsilon= \pm \frac{\mu V_{0}}{\omega} n
$$

We employ the latter in Eq. (20), whereupon the condition $M_{i j} E^{j}=0$ yields

$$
\mathbf{E}_{ \pm}=\frac{1}{\sqrt{2} n \sqrt{n^{2}-n_{1}^{2}}}\left(\begin{array}{c}
n^{2}-n_{1}^{2} \\
\mp \mathrm{i} n_{3} n-n_{1} n_{2} \\
\pm \mathrm{i} n_{2} n-n_{1} n_{3}
\end{array}\right) .
$$

Considering the special choice

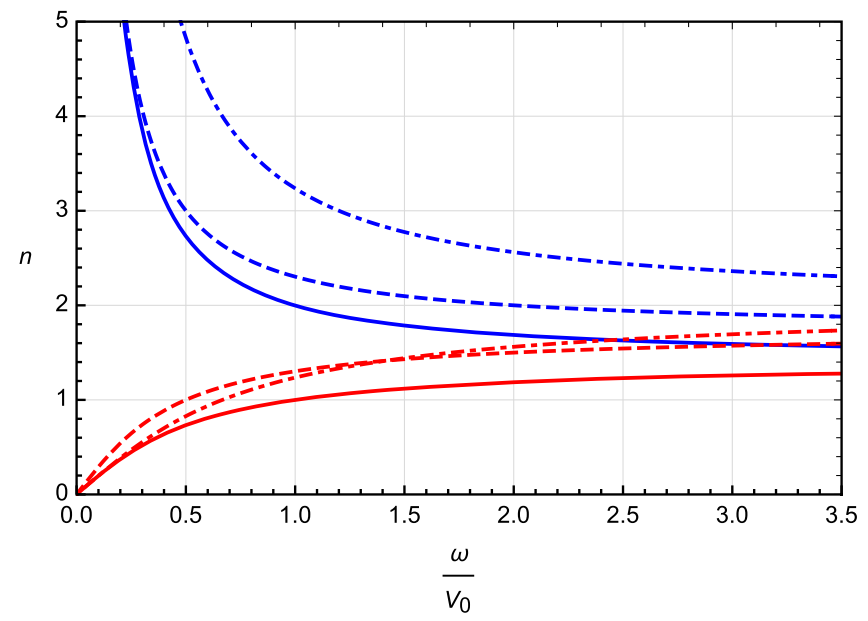

FIG. 1. Refractive indices $n_{ \pm}(\omega)$ of Eq. (28b) in terms of $\omega / V_{0}$. Blue (monotonically decreasing) lines represent $n_{+}$, while red (monotonically increasing) lines depict $n_{-}$. For solid lines, $\mu=1$ and $\epsilon=2$; for dashed lines, $\mu=1$ and $\epsilon=3$; for dashed-dotted lines, $\mu=2$ and $\epsilon=2$. 


$$
\mathbf{n}=\left(\begin{array}{c}
0 \\
0 \\
n_{3}
\end{array}\right)
$$

the normalized electric fields obtained from Eq. (30) are

$$
\mathbf{E}_{ \pm}=\frac{1}{\sqrt{2}}\left(\begin{array}{c}
1 \\
\mp \mathrm{i} \\
0
\end{array}\right)
$$

A polarization is defined to be right-handed (left-handed) if the polarization vector of a plane wave rotates along a circle in clockwise (counterclockwise) direction when the observer is facing into the incoming wave [1,2]. Therefore, $\mathbf{E}_{-}$is interpreted as a left-handed and $\mathbf{E}_{+}$as a right-handed circular polarization vector, respectively. These are associated with the distinct refractive indices $n_{-}$and $n_{+}$of Eq. (28b) that imply different phase velocities of the physical modes giving rise to a rotation of the polarization plane of a linearly polarized wave. The implied birefringence is measured by the specific rotatory power [see the definition of Eq. (8) and Appendix B], here written as

$$
\delta=-\frac{\mu V_{0}}{2},
$$

which is a frequency-independent result dependent on the timelike component $V_{0}$ of the LV background. This nondispersive rotatory power differs from the rotatory power of a typical birefringent crystal, which increases with the frequency, as indicated by Eq. (8) for constant refractive indices. As the refractive indices of Eq. (28b) are real, there is no optical dichroism caused by $V_{0}$.

\section{B. Purely spacelike case}

For the purely spacelike case, $V^{\mu}=(0, \mathbf{V})$, that is, $V^{0}=$ 0 and $\mathbf{V} \neq 0$, Eq. (21) yields

$$
\epsilon\left(n^{2}-\mu \epsilon\right)^{2}-\frac{\mu}{\omega^{2}}\left[\mu \epsilon \mathbf{V}^{2}-n^{2} \mathbf{V}^{2}+(\mathbf{n} \cdot \mathbf{V})^{2}\right]=0 .
$$

Implementing $\mathbf{n} \cdot \mathbf{V}=n|\mathbf{V}| \cos \theta$, one finds

$$
\left(n^{2}-\mu \epsilon\right)^{2}-\frac{\mu^{2}}{\omega^{2}}|\mathbf{V}|^{2} \alpha^{2}=0,
$$

where we defined

$$
\alpha^{2} \equiv 1-\frac{n^{2}}{\mu \epsilon} \sin ^{2} \theta
$$

The two refractive indices (squared) read

$$
\begin{aligned}
n_{ \pm}^{2}= & \mu \epsilon-\frac{\mu \mathbf{V}^{2}}{2 \epsilon \omega^{2}} \sin ^{2} \theta \\
& \pm \frac{\mu|\mathbf{V}|}{2 \epsilon \omega^{2}} \sqrt{4 \epsilon^{2} \omega^{2} \cos ^{2} \theta+\mathbf{V}^{2} \sin ^{4} \theta}
\end{aligned}
$$

It is useful to analyze two special configurations: (i) the perpendicular case where $\mathbf{n} \cdot \mathbf{V}=0$ and $\sin ^{2} \theta=1$, (ii) the longitudinal case where $\mathbf{n} \cdot \mathbf{V}= \pm n|\mathbf{V}|$ and $\sin ^{2} \theta=0$.

In order to examine the propagation modes, let us choose coordinates such that Eq. (31) holds, whereupon Eq. (20) simplifies as

$$
\left[M_{i j}\right]=\left(\begin{array}{ccc}
n^{2}-\mu \epsilon & \mathrm{i} \frac{\mu}{\omega} V_{3} & -\mathrm{i} \frac{\mu}{\omega} V_{2} \\
-\mathrm{i} \frac{\mu}{\omega} V_{3} & n^{2}-\mu \epsilon & \mathrm{i} \frac{\mu}{\omega} V_{1} \\
\mathrm{i} \frac{\mu}{\omega} V_{2} & -\mathrm{i} \frac{\mu}{\omega} V_{1} & -\mu \epsilon
\end{array}\right) .
$$

Solving $M_{i j} E^{j}=0$, one obtains

$$
\mathbf{E}_{ \pm}=E_{0}\left(\begin{array}{c}
V_{1} V_{2}-\mathrm{i} \epsilon \omega V_{3} \\
V_{2}^{2}-\epsilon^{2} \omega^{2} f\left(\alpha_{ \pm}\right) \\
V_{2} V_{3}+\mathrm{i} V_{1} \epsilon \omega f\left(\alpha_{ \pm}\right)
\end{array}\right)
$$

where

$$
f(\alpha)=1+\frac{\alpha^{2}-1}{\sin ^{2} \theta}, \quad \alpha_{ \pm}^{2}=1-\frac{n_{ \pm}^{2}}{\mu \epsilon} \sin ^{2} \theta,
$$

and $E_{0}$ is an appropriate normalization.

\section{V-perpendicular configuration}

Considering the perpendicular configuration with $\sin ^{2} \theta=1$, the solutions of Eq. (34) for $n^{2}$ according to Eq. (36) are

$$
n_{ \pm}^{2}=\mu \epsilon+\frac{\mu \mathbf{V}^{2}}{2 \epsilon \omega^{2}}(-1 \pm 1),
$$

that is

$$
n_{+}=\sqrt{\mu \epsilon}, \quad n_{-}=\sqrt{\mu \epsilon-\frac{\mu \mathbf{V}^{2}}{\epsilon \omega^{2}}} .
$$

While $n_{+}$is the standard refractive index of Maxwell electrodynamics in media, corresponding to $\alpha_{+}=0$, the refractive index $n_{-}$is associated with $\alpha_{-}=|\mathbf{V}| /(\epsilon \omega)$, whereupon it is affected by the background. For $\omega<\omega_{-}$, we have $n_{-}^{2}<0$ and $n_{-}$becomes purely imaginary, so that the corresponding mode no longer propagates. This defines the cutoff frequency,

$$
\omega_{-}=\frac{|\mathbf{V}|}{\epsilon} .
$$




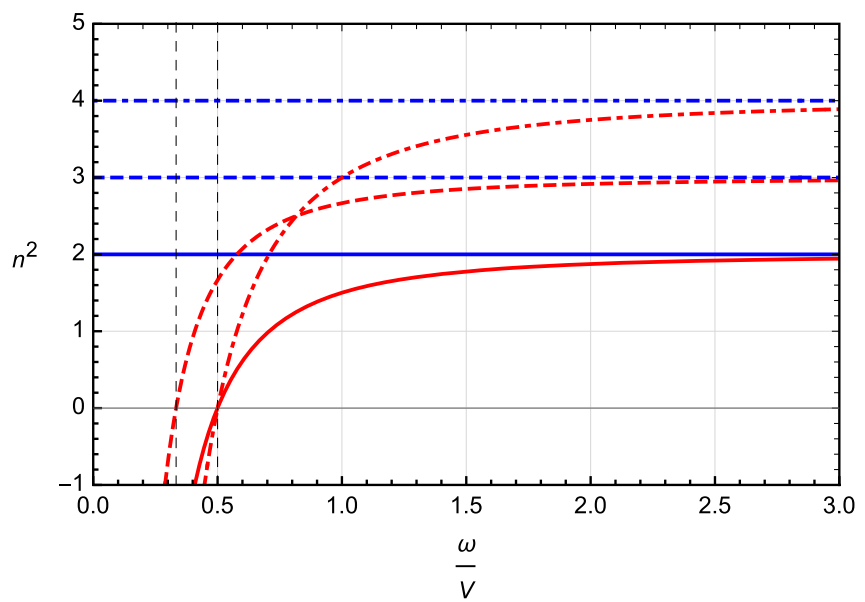

FIG. 2. Refractive indices $n_{ \pm}^{2}(\omega)$ of Eq. (39) in terms of $\omega / V$ where $V=|\mathbf{V}|$. Blue (horizontal) lines represent $n_{+}^{2}$, while red (curved) lines depict $n_{-}^{2}$. For solid lines, $\mu=1$ and $\epsilon=2$; for dashed lines, $\mu=1$ and $\epsilon=3$; for dashed-dotted lines, $\mu=2$ and $\epsilon=2$.

The general behavior of the refractive indices is depicted in Fig. 2, where the squared refractive indices (39) are plotted in terms of the dimensionless parameter $\omega /|\mathbf{V}|$. The horizontal dashed lines stand for $n_{+}^{2}$, which is constant for all frequencies. As for the mode associated with $n_{-}^{2}$, the vertical gray dashed lines (located at different $\omega_{-} /|\mathbf{V}|$ for each case) separate the absorption regime, $\omega<\omega_{-}$, from the propagation regime, $\omega>\omega_{-}$. Furthermore, in the limit of high frequencies, $n_{-}^{2} \mapsto n_{+}^{2}=\mu \epsilon$.

In order to examine the propagation modes, let us choose coordinates such that Eq. (31) holds. Then, a perpendicular background configuration is $\mathbf{V}=\left(V_{1}, V_{2}, 0\right)$. Due to $\alpha_{+}=$ 0 and $f\left(\alpha_{+}\right)=0$, Eq. (38) yields a linearly polarized, transverse mode,

$$
\mathbf{E}_{+}=\frac{1}{|\mathbf{V}|}\left(\begin{array}{c}
V_{1} \\
V_{2} \\
0
\end{array}\right) \equiv \hat{\mathbf{V}}
$$

where $\hat{\mathbf{V}}$ is a unit vector pointing along the direction of $\mathbf{V}$. Also, inserting $\alpha_{-}$into Eq. (38) provides another linearly polarized mode that has an additional longitudinal component:

$$
\begin{aligned}
\mathbf{E}_{-} & =E_{0}^{(-)}\left(\begin{array}{c}
V_{2} \\
-V_{1} \\
\mathrm{i}\left(V_{1}^{2}+V_{2}^{2}\right) /(\epsilon \omega)
\end{array}\right) \\
& =\tilde{E}_{0}^{(-)}\left(\hat{\mathbf{V}} \times \hat{\mathbf{n}}+\mathrm{i} \frac{|\mathbf{V}|}{\epsilon \omega} \hat{\mathbf{n}}\right),
\end{aligned}
$$

where $E_{0}^{(-)}, \tilde{E}_{0}^{(-)}$are properly chosen amplitudes and $\hat{\mathbf{n}}$ is the unit vector pointing along the propagation direction of
Eq. (31). Note that the longitudinal component is suppressed by the magnitude of the preferred direction $\mathbf{V}$ in comparison to the transverse part. For $V_{2}=0$ the behavior is even more transparent:

$$
\mathbf{E}_{+}=\left(\begin{array}{l}
1 \\
0 \\
0
\end{array}\right), \quad \mathbf{E}_{-}=\tilde{E}_{0}^{(-)}\left(\begin{array}{c}
0 \\
-1 \\
\mathrm{i} V_{1} /(\epsilon \omega)
\end{array}\right) .
$$

The structure of Eqs. (42), (43) reveals immediately that $\mathbf{E}_{+} \cdot \mathbf{E}_{-}^{*}=\mathbf{E}_{+}^{*} \cdot \mathbf{E}_{-}=0$, i.e., both polarization vectors are orthogonal to each other. The refractive indices (40) are associated with the linearly polarized modes of Eq. (44). Although the vector $\mathbf{E}_{-}$is composed of a transverse and a longitudinal component, as for polarization properties, it is interpreted as a linearly polarized mode and only its transverse component is taken into account.

If birefringence originates from two linearly polarized modes having different phase velocities, this property is not suitably characterized in terms of the usual rotatory power given by Eq. (8). Note that the latter is based on a decomposition of a linearly polarized mode into two circularly polarized ones of different chirality (see Appendix B). Instead, in the propagation regime, $\omega>\omega_{-}$, the phase shift developed between the propagating modes as a consequence of the distinct phase velocities is valuable to characterize birefringence (see Eq. (8.32) in [6]):

$$
\Delta=\frac{2 \pi}{\lambda_{0}} d\left(n_{+}-n_{-}\right) .
$$

Here, $\lambda_{0}$ is the wavelength of the electromagnetic radiation in vacuo and $d$ corresponds to the thickness of the medium or the distance the wave travels in the medium. Starting from the refractive indices of Eq. (40), the phase shift per unit length is

$$
\frac{\Delta}{d}=\frac{2 \pi}{\lambda_{0}} \sqrt{\mu \epsilon}\left(1-\sqrt{1-\frac{\mathbf{V}^{2}}{\epsilon^{2} \omega^{2}}}\right),
$$

which simplifies to

$$
\frac{\Delta}{d} \simeq \frac{\pi \sqrt{\mu \epsilon}}{\lambda_{0} \epsilon^{2} \omega^{2}} \mathbf{V}^{2},
$$

in the limit $|\mathbf{V}| / \omega \ll 1$. Notice that $n_{-}$is real for $\omega>\omega_{-}$or $|\mathbf{V}| / \omega<\epsilon$, so that in the limit $|\mathbf{V}| / \omega \ll 1$, the expression (46) remains real, justifying the result (47). These findings indicate that birefringence is governed by the norm squared of the LV background vector $\mathbf{V}$ and depends quadratically on the inverse of the frequency $\omega$, as well. That dependence is neither observed in the purely timelike case (see Eq. (33) for comparison) nor in usual crystals [see Eq. (45)]. 
For $\omega<\omega_{-}, n_{-}$becomes complex while $n_{+}$remains real. Thus, absorption (only) occurs for the mode labeled with a minus sign. In this case, the absorption coefficient [2], $\gamma=2 \omega \operatorname{Im}(n)$, reads

$$
\gamma=2 \sqrt{\mu \epsilon} \omega \sqrt{-1+\frac{|\mathbf{V}|^{2}}{\omega^{2} \epsilon^{2}}} .
$$

So the mode associated with Eq. (43) is absorbed, whereas the remaining mode given by Eq. (42) propagates without attenuation. Therefore, after traveling a certain distance in such a medium, only the mode of Eq. (42) will survive.

\section{V-longitudinal configuration}

We now consider configurations where $\mathbf{n} \cdot \mathbf{V}= \pm n|\mathbf{V}|$ implying $\sin ^{2} \theta=0$ and $\alpha^{2}=1$ in Eq. (35a). This means that $\mathbf{n}$ and $\mathbf{V}$ point along the same direction, i.e., for $\mathbf{n}$ given by Eq. (31) we choose $\mathbf{V}=\left(0,0, V_{3}\right)$. Hence, based on Eq. (36), the solutions of Eq. (35a) for $n^{2}$ in this case are

$$
n_{ \pm}^{2}=\mu \epsilon \pm \frac{\mu|\mathbf{V}|}{\omega}
$$

Note that $n_{+}^{2}>0$, meaning that the mode associated with the refractive index $n_{+}$propagates within the full frequency domain. On the other hand, the mode associated with $n_{-}$ just propagates for $\omega>\omega_{-}$, for which $n_{-}^{2}>0$. Here, $\omega_{-}$is the cutoff frequency of Eq. (41). This description is verified in Fig. 3, where the refractive indices (49) are depicted as functions of the dimensionless parameter $\omega /|\mathbf{V}|$. The modes associated with $n_{+}^{2}$ and $n_{-}^{2}$ exhibit anomalous and

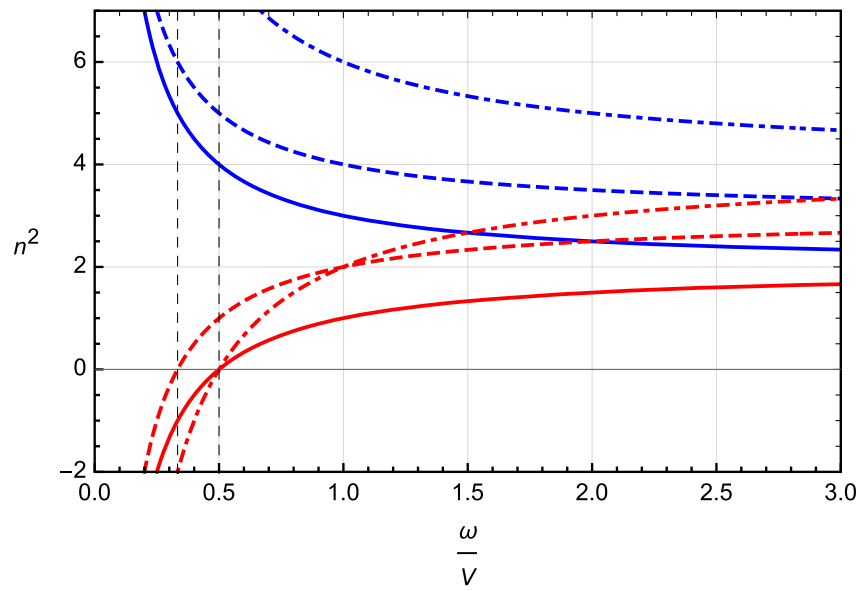

FIG. 3. Refractive indices $n_{ \pm}^{2}(\omega)$ of Eq. (49) in terms of $\omega / V$ where $V=|\mathbf{V}|$. Blue (monotonically decreasing) lines represent $n_{+}^{2}$, while red (monotonically increasing) lines depict $n_{-}^{2}$. For solid lines, $\mu=1$ and $\epsilon=2$; for dashed lines, $\mu=1$ and $\epsilon=3$; for dotted-dashed lines, $\mu=2$ and $\epsilon=2$. The vertical dashed lines, from left to right, are given by $\omega_{-} / V=1 / 3$ and $\omega_{-} / V=1 / 2$, respectively, with $\omega_{-}$from Eq. (41). normal dispersion, respectively, recovering the standard value $n_{ \pm}^{2} \rightarrow \mu \epsilon$ in the regime of high frequencies.

For the special choice of Eq. (31), $M_{i j} E^{j}=0$ provides

$$
\mathbf{E}_{ \pm}=\frac{1}{\sqrt{2}}\left(\begin{array}{c}
1 \\
\pm \mathrm{i} \\
0
\end{array}\right) .
$$

Here, $\mathbf{E}_{+}$and $\mathbf{E}_{-}$represent polarization vectors for lefthanded and right-handed circularly polarized modes, respectively. The two refractive indices of Eq. (49) also imply birefringence providing the following rotatory power:

$$
\delta=-\frac{\sqrt{\mu \epsilon}}{2} \omega\left(\sqrt{1+\frac{|\mathbf{V}|}{\omega \epsilon}}-\sqrt{1-\frac{|\mathbf{V}|}{\omega \epsilon}}\right) .
$$

In the limit $|\mathbf{V}| / \omega \ll 1$, the quantity $n_{-}$remains real, which also implies a real rotatory power,

$$
\delta \simeq-\frac{1}{2} \sqrt{\frac{\mu}{\epsilon}}|\mathbf{V}|,
$$

representing frequency-independent birefringence, similarly to Eq. (33).

On the other hand, for $|\mathbf{V}| / \omega>\epsilon, n_{-}$becomes purely imaginary, while $n_{+}$remains real. In this frequency regime, both modes are absorbed to a different degree. The latter is characterized by the dichroism coefficient defined in Eq. (9), which yields (cf. Eq. (48)):

$$
\delta_{\mathrm{d}}=\frac{\sqrt{\mu \epsilon}}{2} \omega \sqrt{-1+\frac{|\mathbf{V}|}{\omega \epsilon}} .
$$

With the latter finding at hand, we bring our study of the essential properties of MCFJ theory in continuous media to a close.

\section{HIGHER-DERIVATIVE DIMENSION-FIVE ELECTRODYNAMICS IN MATTER}

After analyzing the properties of MCFJ theory in a material [see Eq. (11)], the next logical step is to construct and investigate an extension involving additional fourderivatives. Such extensions are naturally contained in the nonminimal (nongravitational) SME [69-71], which is a comprehensive framework for the parametrization of Lorentz and $C P T$ violation in effective field theory in Minkowski spacetime. For the past two decades it has been the foundation of various experiments testing the fundamental spacetime symmetries [91]. No signal of Lorentz violation in vacuo has been found, so far. However, Lorentz violation can be considered as an intrinsic property of material media, which is why the SME is more than 
suitable as a base for representing certain material properties within a field theory setting and to even propose novel materials with unusual characteristics.

The electromagnetic sector of the nonminimal SME gives rise to a modified electrodynamics and is given by

$$
\begin{aligned}
\mathcal{L}= & -\frac{1}{4} F_{\mu \nu} F^{\mu \nu}+\frac{1}{2} \epsilon^{\kappa \lambda \mu \nu} A_{\lambda}\left(\hat{k}_{A F}\right)_{\kappa} F_{\mu \nu} \\
& -\frac{1}{4} F_{\kappa \lambda}\left(\hat{k}_{F}\right)^{\kappa \lambda \mu \nu} F_{\mu \nu} .
\end{aligned}
$$

The $C P T$-odd and $C P T$-even operators, $\left(\hat{k}_{A F}\right)_{\kappa}$ and $\left(\hat{k}_{F}\right)^{\kappa \lambda \mu \nu}$, respectively, are the analogs of $\left(k_{A F}\right)_{\kappa}$ and $\left(k_{F}\right)^{\kappa \lambda \mu \nu}$ of the minimal SME. However, they involve nonminimal coefficients contracted with additional fourderivatives in the form of the following infinite operator series:

$$
\begin{aligned}
\left(\hat{k}_{A F}\right)_{\kappa} & =\sum_{d \text { odd }}\left(k_{A F}^{(d)}\right)_{\kappa} \alpha_{1} \ldots \alpha_{(d-3)} \partial_{\alpha_{1}} \ldots \partial_{\alpha_{(d-3)}}, \\
\left(\hat{k}_{F}\right)^{\kappa \lambda \mu \nu} & =\sum_{d \text { even }}\left(k_{F}^{(d)}\right)^{\kappa \lambda \mu \nu \alpha_{1} \ldots \alpha_{(d-4)}} \partial_{\alpha_{1}} \ldots \partial_{\alpha_{(d-4)}},
\end{aligned}
$$

where $d$ is the mass dimension of the tensor field operator that a certain coefficient is contracted with. Besides, $(4-d)$ is the mass dimension of the associated controlling coefficients $\left(k_{A F}^{(d)}\right)_{\kappa}^{\alpha_{1} \ldots \alpha_{(d-3)}}$ and $\left(k_{F}^{(d)}\right)^{\kappa \lambda \mu \nu \alpha_{1} \ldots \alpha_{(d-4)}}$. The Lorentz indices $\alpha_{i}$ are contracted with additional spacetime derivatives.

We are interested in the $C P T$-odd dimension-five $(d=5)$ extension, which is represented by a CFJ-like term of the form

$$
\frac{1}{2} \epsilon^{\kappa \lambda \mu \nu} A_{\lambda}\left(\hat{k}_{A F}\right)_{\kappa} F_{\mu \nu}
$$

with

$$
\left(\hat{k}_{A F}\right)_{\kappa}=\left(k_{A F}^{(5)}\right)_{\kappa}^{\alpha_{1} \alpha_{2}} \partial_{\alpha_{1}} \partial_{\alpha_{2}} .
$$

For our investigation, we will use the parametrization

$$
\left(k_{A F}^{(5)}\right)_{\kappa}^{\alpha_{1} \alpha_{2}}=U_{\kappa} \eta^{\alpha_{1} \alpha_{2}},
$$

with the Lorentz-violating four-vector, $U_{\kappa}$, and the Minkowski metric tensor, $\eta^{\mu \nu}$. Using Eq. (57), the higher-derivative term becomes

$$
\frac{1}{2} \epsilon^{\kappa \lambda \mu \nu} A_{\lambda} U_{\kappa} \square
$$

where we have introduced the d'Alembertian $\square=$ $\eta^{\alpha_{1} \alpha_{2}} \partial_{\alpha_{1}} \partial_{\alpha_{2}}$. The resulting higher-derivative Lagrangian,

$$
\mathcal{L}=-\frac{1}{4} F^{\mu \nu} F_{\mu \nu}+\frac{1}{2} \epsilon^{\beta \lambda \mu \nu} U_{\beta} A_{\lambda} \square F_{\mu \nu}-A_{\mu} J^{\mu},
$$

involves LV parametrized by the background vector, $U^{\mu}=\left(U^{0}, \mathbf{U}\right)$. Some classical aspects of this model were examined in Refs. [73,75].

In order to study the effects of this higher-derivative term on electromagnetic propagation in continuous matter, we take as a starting point the Lagrangian (59), but employ the field strength tensor $G^{\mu \nu}$ in its kinetic term, as it occurs in Eq. (2a). Thus, the Lagrangian of this new model is

$$
\mathcal{L}=-\frac{1}{4} G^{\mu \nu} F_{\mu \nu}+\frac{1}{2} \epsilon^{\beta \lambda \mu \nu} U_{\beta} A_{\lambda} \square F_{\mu \nu}-A_{\mu} J^{\mu},
$$

where the tensor $G^{\mu \nu}$ is written in terms of the constitutive tensor $\chi^{\mu \nu \alpha \beta}$, defined in Eqs. (2b), (3). The latter provides a generalization of the electrodynamics of Eq. (59) in matter. One may expect a connection between this theory and a generalization of the modified Dirac theory given by Eq. (10) where additional derivatives are included in the second contribution. However, it is beyond the scope of the current paper to demonstrate such a connection explicitly. Thus, by using Eq. (59) we can take into consideration an additional energy-momentum dependence that goes beyond that of the CFJ term in matter.

The Lagrangian of Eq. (60) involves a third-order derivative of the four-potential, which requires an associated Euler-Lagrange equation endowed with derivatives for field derivatives that are of the same order. In principle, the derivative order can be decreased by rewriting Eq. (60) in the form

$\mathcal{L}=-\frac{1}{4} G^{\mu \nu} F_{\mu \nu}-\frac{1}{2} \epsilon^{\beta \lambda \mu \nu} U_{\beta}\left(\partial_{\eta} A_{\lambda}\right) \partial^{\eta} F_{\mu \nu}-A_{\mu} J^{\mu}$.

As for the Lagrangian of Eq. (61), it is enough to consider the Euler-Lagrange equation involving derivatives for second-order derivatives of the fields, that is,

$$
\frac{\partial \mathcal{L}}{\partial A_{\kappa}}-\partial_{\rho}\left(\frac{\partial \mathcal{L}}{\partial\left(\partial_{\rho} A_{\kappa}\right)}\right)+\partial_{\alpha} \partial_{\rho}\left(\frac{\partial \mathcal{L}}{\partial\left(\partial_{\rho} \partial_{\alpha} A_{\kappa}\right)}\right)=0 .
$$

Applying the latter to Eq. (61) yields

$$
\partial_{\rho} G^{\rho \kappa}+\epsilon^{\beta \kappa \mu \nu} U_{\beta} \square F_{\mu \nu}=J^{\kappa} .
$$

In this scenario, the modified Gauss's and Ampère's laws are

$$
\begin{array}{r}
\nabla \cdot \mathbf{D}+2 \square(\mathbf{U} \cdot \mathbf{B})=\rho, \\
\nabla \times \mathbf{H}-\partial_{t} \mathbf{D}+2 \square U_{0} \mathbf{B}-2 \square(\mathbf{U} \times \mathbf{E})=\mathbf{J},
\end{array}
$$

respectively. These modified inhomogeneous Maxwell equations can describe new effects on the propagation of 
TABLE II. Behavior of the LV terms in the Lagrangian of Eq. (59) under charge conjugation, parity transformation, and time reversal.

\begin{tabular}{cccccccc}
\hline \hline & $\mathbf{E}$ & $\mathbf{B}$ & $A_{0}$ & $\mathbf{A}$ & $U_{0}(\mathbf{A} \cdot \square \mathbf{B})$ & $A_{0}(\mathbf{U} \cdot \square \mathbf{B})$ & $\mathbf{U} \cdot(\mathbf{A} \times \square \mathbf{E})$ \\
\hline$C$ & - & - & - & - & + & + & + \\
$P$ & - & + & + & - & - & + & + \\
$T$ & + & - & + & - & + & - & - \\
\hline \hline
\end{tabular}

electromagnetic waves in continuous media characterized by the constitutive tensor $\chi^{\mu \nu \alpha \beta}$. In the forthcoming sections we obtain the dispersion relations and study the behavior of refractive indices and propagating modes for a medium characterized by the usual constitutive relations, $\mathbf{D}=\epsilon \mathbf{E}$ and $\mathbf{H}=\mu^{-1} \mathbf{B}$.

With regards to the discrete symmetries, the background $U^{\mu}$ in the Lagrangian of Eq. (60) behaves in the very exact way as $V^{\mu}$ does in Eq. (11), since the two $C P T$-odd terms differ from each other by the presence of the second-order differential operator, $\square$, that is even under the discrete symmetries $P$ and $T$. In fact, by simple inspection, one finds that the terms involving the timelike coefficient, $U_{0}$, are $P$-odd, $C$-even, $T$-even, and $P T$-odd, while the contributions with $\mathbf{U}$ are $P$-even, $C$-even, $T$-odd, and $P T$-odd, as shown in Table II. This means that the terms proportional to $U_{0}$ and $\mathbf{U}$ will act as a source for optical activity (as well as birefringence) of the medium under study.

\section{A. Dispersion relations}

As is commonly known, the Maxwell equations constitute one starting point for achieving the dispersion relations in electrodynamics. Taking the time derivative of Eq. (64b) and employing Eq. (6d), one obtains

$\partial_{t} \nabla \times \mathbf{H}-\partial_{t}^{2} \mathbf{D}-2 \square U_{0}(\nabla \times \mathbf{E})-2 \partial_{t} \square(\mathbf{U} \times \mathbf{E})=\partial_{t} \mathbf{J}$.

Using now the constitutive relations given in Eqs. (15) and (16) as well as $\mathbf{J}=\sigma \mathbf{E}$ and the plane-wave ansatz for the fields, Eq. (65) yields

$$
\left[\mathbf{k}^{2} \delta_{i j}-k_{i} k_{j}-\omega^{2} \mu \bar{\epsilon}_{i j}(\omega)\right] E^{j}=0,
$$

where we have defined the effective permittivity tensor [cf. Eq. (18b)]

$$
\begin{aligned}
\bar{\epsilon}_{i j}(\omega) \equiv & \left(\epsilon+\mathrm{i} \frac{\sigma}{\omega}\right) \delta_{i j}-\frac{2 \mathrm{i}}{\omega^{2}}\left(k^{2}-\omega^{2}\right) \\
& \times \epsilon_{i a j}\left(\omega U_{a}-k_{a} U_{0}\right) .
\end{aligned}
$$

The latter quantity is interpreted as an extended frequencydependent electric permittivity, which contains contributions stemming from the higher-derivative term. On the right-hand side of Eq. (66b), the contribution involving $\epsilon_{i a j} \omega U_{a}$ violates time reversal invariance, while the term $\epsilon_{i a j} k_{a} U_{0}$ breaks parity invariance. Using $\mathbf{k}=\omega \mathbf{n}$, Eq. (66a) can now be cast into the form:

$$
M_{i j} E^{j}=0,
$$

with the tensor $M_{i j}$ given by

$$
M_{i j}=n^{2} \delta_{i j}-n_{i} n_{j}-\mu \bar{\epsilon}_{i j}(\omega),
$$

while the effective permittivity tensor now reads

$$
\begin{aligned}
\bar{\epsilon}_{i j}(\omega)= & \left(\epsilon+\mathrm{i} \frac{\sigma}{\omega}\right) \delta_{i j}-2 \mathrm{i} \omega\left(n^{2}-1\right) \\
& \times \epsilon_{i a j}\left(U_{a}-n_{a} U_{0}\right) .
\end{aligned}
$$

It is important to note that although the medium has an isotropic electric permittivity $\epsilon \delta_{i j}$, anisotropy effects are generated by the background $U_{\mu}$, present in the off-diagonal components of $\bar{\epsilon}_{i j}(\omega)$ in Eq. (67c).

The matrix $M_{i j}$ in Eq. (67b) has the explicit form

$$
\left[M_{i j}\right]=\mathcal{M}+2 \mathrm{i} \mu \omega\left(n^{2}-1\right) \mathcal{W}
$$

with $\mathcal{M}$ given by Eq. (20b) and

$\mathcal{W}=\left(\begin{array}{ccc}0 & U_{0} n_{3}-U_{3} & U_{2}-U_{0} n_{2} \\ U_{3}-U_{0} n_{3} & 0 & U_{0} n_{1}-U_{1} \\ U_{0} n_{2}-U_{2} & U_{1}-U_{0} n_{1} & 0\end{array}\right)$

Evaluating $\operatorname{det}\left[M_{i j}\right]=0$ implies the dispersion equation

$$
\begin{aligned}
0= & \tilde{\epsilon}\left(n^{2}-\mu \tilde{\epsilon}\right)^{2}-4\left(n^{2}-1\right)^{2} \mu \omega^{2} \\
& \times\left\{\mu \tilde{\epsilon}\left[U_{0}^{2} n^{2}+\mathbf{U}^{2}-2 U_{0}(\mathbf{n} \cdot \mathbf{U})\right]-\mathbf{U}^{2} \mathbf{n}^{2}+(\mathbf{n} \cdot \mathbf{U})^{2}\right\},
\end{aligned}
$$

with $\tilde{\epsilon}$ stated in Eq. (21b). We point out that by employing the four-momentum of Eq. (22) as well as

$$
\bar{U}^{\mu} \equiv\left(\sqrt{\mu} U^{0}, \frac{\mathbf{U}}{\sqrt{\tilde{\epsilon}}}\right)
$$

we can cast the dispersion equation into the form

$$
\bar{p}^{4}+4 p^{4}\left[\bar{U}^{2} \bar{p}^{2}-(\bar{U} \cdot \bar{p})^{2}\right]=0 .
$$

By consulting the effective metric of Eq. (24), the latter can also be expressed in terms of the conventional fourmomentum $p^{\mu}$ and the preferred direction $U^{\mu}$ as follows: 


$$
\begin{aligned}
0= & (p \cdot \tilde{\eta} \cdot p)^{2}+4(p \cdot \eta \cdot p)^{2} \frac{\mu}{\tilde{\epsilon}} \\
& \times\left[(U \cdot \tilde{\eta} \cdot U)(p \cdot \tilde{\eta} \cdot p)-(U \cdot \tilde{\eta} \cdot p)^{2}\right] .
\end{aligned}
$$

Note that in contrast to the dispersion equation of MCFJ theory stated in Eq. (23), the recent Eq. (71) cannot be written in terms of the effective four-momentum $\bar{p}^{\mu}$ only, but $p^{\mu}$ is necessary, as well. The reason for $p^{\mu}$ playing a role are the two additional four-derivatives contracted with the dimension-5 coefficients in Eq. (57). Equation (72) also allows us to say that the propagation of modified electromagnetic waves in media is governed by two metrics: the Minkowski metric $\eta_{\mu \nu}$ and the effective metric $\tilde{\eta}_{\mu \nu}$ of Eq. (24). Thus, the dimension-5 MCFJ-type theory defined by Eq. (60) could be called bimetric in this sense. We conclude that the structure of the dimension-5 MCFJ-type theory in media is quite different from that of the generalized MCFJ model in Eq. (60).

In vacuo, the constitutive parameters read $\epsilon=1, \mu=1$, and $\sigma=0$. In this case, the dispersion equation in Eq. (69) reduces to

$$
\begin{aligned}
0= & \left(n^{2}-1\right)^{2}\left\{1-4 \omega^{2}\left[U_{0}^{2} n^{2}-\mathbf{U}^{2} n^{2}+\mathbf{U}^{2}\right.\right. \\
& \left.\left.+(\mathbf{n} \cdot \mathbf{U})^{2}-2 U_{0}(\mathbf{n} \cdot \mathbf{U})\right]\right\},
\end{aligned}
$$

being conveniently simplified as

$$
p^{4}\left\{1+4\left[p^{2} U^{2}-(U \cdot p)^{2}\right]\right\}=0,
$$

with the four-momentum $p^{\mu}$ and the preferred direction $U^{\mu}=\left(U^{0}, \mathbf{U}\right)$. Notice that Eq. (74) recovers the dispersion equation obtained in Eq. (23) of Ref. [75], where this higher-derivative electrodynamics was examined in vacuo. It is important to point out that the remarkable difference between Eq. (71) and Eq. (74) is ascribed to the presence of the continuous medium, since the dimension-five higherderivative terms in the Lagrangians of Eqs. (59), (60) correspond to each other.

In what follows, we analyze the dispersion equation (69) for the timelike and spacelike configurations of the vectorial background, $U^{\mu}$.

\section{B. Purely timelike case}

Considering the purely timelike scenario for the background vector, $U_{0} \neq 0$ and $\mathbf{U}=\mathbf{0}$, and also $\tilde{\epsilon} \mapsto \epsilon$, which means that the medium does not have Ohmic conductivity (whereupon $\sigma=0$ ), Eq. (69) is reduced to the form

$$
\epsilon\left(n^{2}-\mu \epsilon\right)^{2}-4 \mu^{2} \omega^{2} U_{0}^{2} \epsilon n^{2}\left(n^{2}-1\right)^{2}=0,
$$

implying

$$
n^{2}-\mu \epsilon= \pm 2 \mu \omega U_{0} n\left(n^{2}-1\right),
$$

TABLE III. Sign of discriminant $\Delta$ of Eq. (76) and the nature of the roots (solutions) of Eq. (75c).

\begin{tabular}{ll}
\hline \hline Sign & Solutions \\
\hline$\Delta>0$ & One real root and two complex conjugate roots \\
$\Delta \leq 0$ & $\begin{array}{c}\text { Three real roots (with two or all three } \\
\text { equal to each other if } \Delta=0)\end{array}$ \\
\hline \hline
\end{tabular}

or equivalently

$$
\pm 2 \mu \omega U_{0} n^{3}-n^{2} \mp 2 \mu \omega U_{0} n+\mu \epsilon=0 .
$$

The latter equation is cubic in $n$ and has 3 (complex) solutions, in general, given as functions $n=n(\omega)$. These solutions extend to frequency domains defined in accordance with the sign of the discriminant of the cubic equation, written as

$$
\Delta=\frac{S}{2^{4} 3^{3} \mu^{3} \omega^{4} U_{0}^{4}},
$$

with

$S=-\epsilon-\mu \omega^{2} U_{0}^{2}\left[1+9 \mu \epsilon(2-3 \mu \epsilon)+16 \mu^{2} \omega^{2} U_{0}^{2}\right]$.

For a cubic polynomial equation, the sign of $\Delta$ helps us to identify the nature (real or complex) of the 3 solutions, in accordance with Table III.

Since the denominator of Eq. (76a) is positive, we only need to analyze the sign of the numerator, $S$. As $S$ is a function quartic in $\omega$, it is possible to find two roots that provide three frequency ranges for positive or negative values of $\Delta$. In this way, the relation $S=0$ establishes the critical values of frequencies (roots) that separate the absorption domain $S>0$ from the propagation domain $S<0$. Solving $S=0$, one achieves two roots for $\omega^{2}$ given by

$\omega_{ \pm}^{2}=\frac{1}{32 \mu^{2} U_{0}^{2}}\left\{9 \mu \epsilon(3 \mu \epsilon-2)-1 \pm \sqrt{\mu \epsilon-1}(9 \mu \epsilon-1)^{3 / 2}\right\}$.

Thus, the three frequency ranges associated with two distinct scenarios are as follows:

(i) For $\omega_{-}<\omega<\omega_{+}$, one has $S>0$ and $\Delta>0$, so that Eq. (75c) yields one real function $n(\omega)$ and two complex functions $n(\omega)$.

(ii) For $\omega<\omega_{-}$or $\omega>\omega_{+}$, one has $S<0$ and $\Delta<0$, so that there are three real refractive indices $n(\omega)$.

The first domain describes absorption effects, whereas electromagnetic waves can freely propagate without attenuation in the second domain. The sign of $S$ determines the real or complex nature of $n(\omega)$ in the corresponding frequency range. For a complex refractive index, we can 


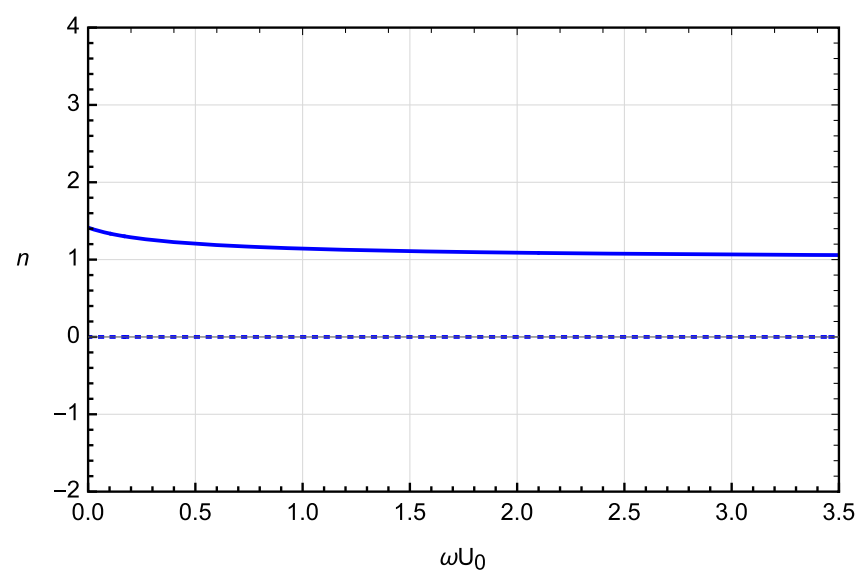

FIG. 4. Plot of one real root of Eq. (75c), the refractive index $n_{1}(\omega)$, in terms of $\omega U_{0}$. It is obtained by choosing the lower signs of Eq. (75c). The solid (dotted) line represents $\operatorname{Re}\left[n_{1}(\omega)\right]$ $\left(\operatorname{Im}\left[n_{1}(\omega)\right]\right)$ where the latter vanishes.

write $n(\omega)=n^{\prime}(\omega)+\mathrm{i} n^{\prime \prime}(\omega)$, where $\operatorname{Re}[n(\omega)]=n^{\prime}(\omega)$ is the refractive index of the medium, and $\operatorname{Im}[n(\omega)]=n^{\prime \prime}(\omega)$ is associated with the medium's absorption coefficient $\alpha=$ $2 \omega n^{\prime \prime}(\omega)[2]$.

Joining the above domains, we can conclude that:

(a) For $\omega<\omega_{-}$there are three real solutions.

(b) For $\omega_{-}<\omega<\omega_{+}$two solutions become complex and the remaining one stays real.

(c) For $\omega>\omega_{+}$the three solutions become real again.

In general, propagation without attenuation is associated with real (positive) refractive indices, whereas absorption effects (damping of the amplitude of electromagnetic waves) are related to complex refractive indices. The modified electrodynamics defined by Eq. (61) ascribes a conducting behavior to a dielectric substrate (with

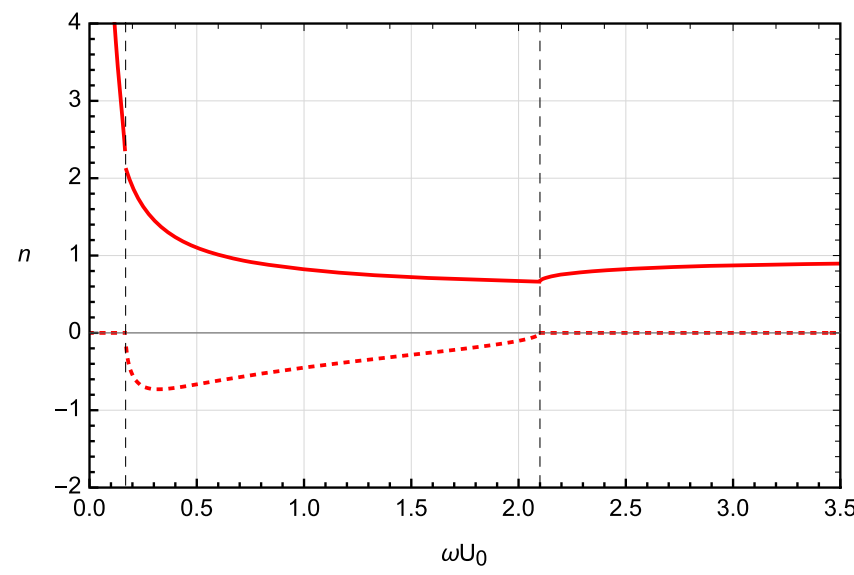

FIG. 5. Plot of one complex root of Eq. (75c), the refractive index $n_{2}(\omega)$, in terms of $\omega U_{0}$. It follows from Eq. (75c) with the upper signs taken into account. The solid (dotted) line represents $\operatorname{Re}\left[n_{2}(\omega)\right]\left(\operatorname{Im}\left[n_{2}(\omega)\right]\right)$.

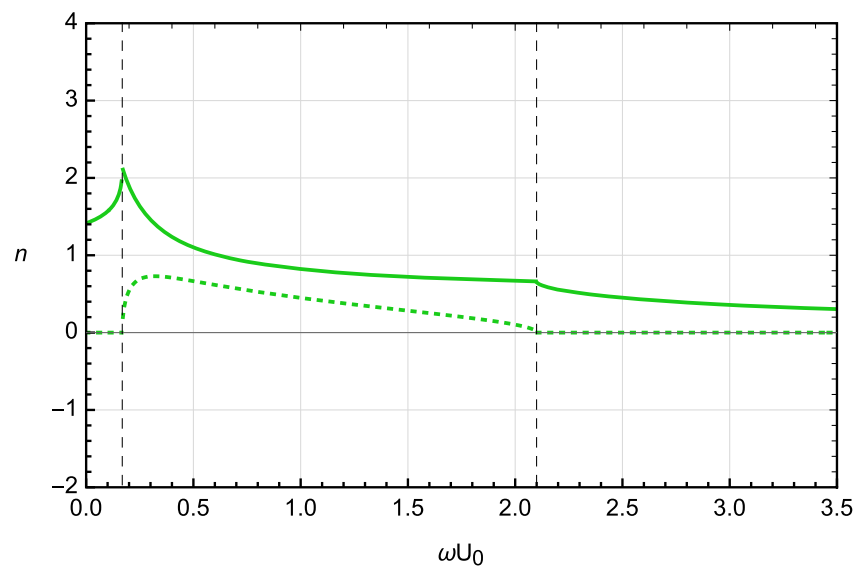

FIG. 6. Plot of one complex root of Eq. (75c), the refractive index $n_{3}(\omega)$, in terms of $\omega U_{0}$. It results from choosing the upper signs of Eq. (75c). The solid (dotted) line depicts $\operatorname{Re}\left[n_{3}(\omega)\right]$ $\left(\operatorname{Im}\left[n_{3}(\omega)\right]\right)$.

additional magnetic properties). For the particular scenario studied previously, electromagnetic waves propagate without being damped in the frequency range where the three solutions are real. In the range where complex solutions for $n(\omega)$ occur, both propagation and absorption (attenuation) is observed. These novel effects stem from the higherderivative coupling of the background coefficient $U_{0}$ with the electromagnetic fields.

The refractive indices for a continuous medium with signal propagation described by Eq. (75c) are given by very intricate expressions [the roots of Eq. (75c)], which will not be stated here explicitly. We depict these three functions, $n_{i}(\omega)$, for $i=1,2,3$ in terms of the dimensionless parameter $\omega U_{0}$ for the special values $\mu=1$ and $\epsilon=2$. These plots are presented in Figs. 4, 5, and 6, where the solid (dotted) lines stand for the real (imaginary) part of $n(\omega)$. The refractive indices shown in the previous figures are characterized by positive real parts. The remaining three refractive indices, which follow from the generic sixthorder polynomial of Eq. (75a), have negative real parts.

We notice that $n_{1}(\omega)$ is real for the full frequency range. The functions $n_{2}(\omega)$ and $n_{3}(\omega)$ become complex in the range $\omega_{-}<\omega<\omega_{+}$, in agreement with the previous analysis. Combining all three plots in Fig. 7, we realize the full scenario described in items (a)-(c) previously stated. The vertical dashed lines indicate the critical frequency values of Eq. (77), namely $\omega_{-} U_{0}$ and $\omega_{+} U_{0}$, which define the transition between the ranges given in (a)-(c). Another characteristic of Figs. 5 and 6 are the discontinuities in the real parts of $n_{2}(\omega)$ and $n_{3}(\omega)$, at the frequencies $\omega_{ \pm}$. Note that $n_{2}(\omega)$ and $n_{3}(\omega)$ become purely imaginary at these values.

As a final comment, we point out that the physical behavior described above only occurs for the higherderivative electrodynamics of Eq. (61) in matter. In fact, in vacuo, Eq. (75a) would provide 


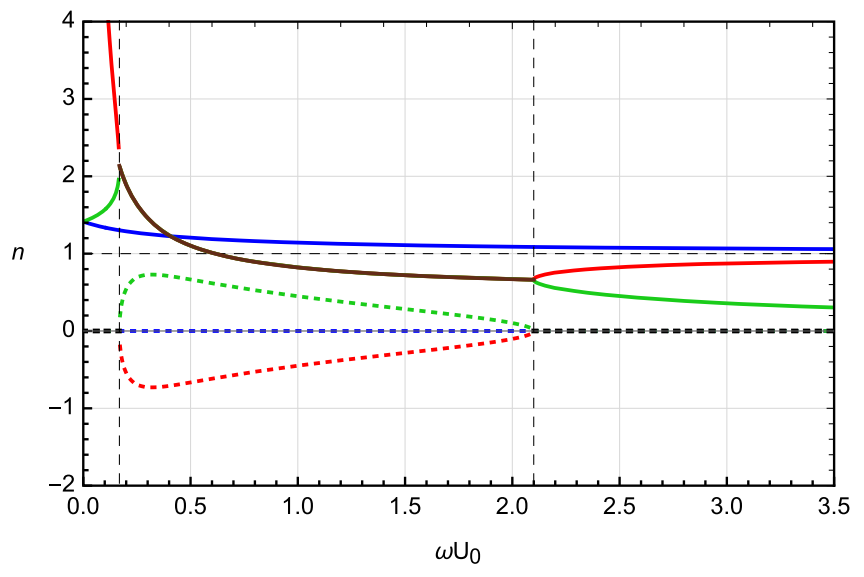

FIG. 7. Compilation of complex refractive indices $n(\omega)$ from Figs. 4, 5, and 6. The solid lines illustrate the real parts of $n_{1}(\omega)$ (blue), $n_{2}(\omega)$ (red), and $n_{3}(\omega)$ (green). Their corresponding imaginary pieces are represented by dotted lines with the same colors. The solid brown line indicates that $\operatorname{Re}\left[n_{2}(\omega)\right]$ and $\operatorname{Re}\left[n_{3}(\omega)\right]$ lie on top of each other. Black dotted lines are employed whenever all three imaginary parts merge.

$$
\left(n^{2}-1\right)^{2}\left(1-4 \omega^{2} U_{0}^{2} n^{2}\right)=0,
$$

whose solutions are real, namely:

$$
n=1, \quad n=\frac{1}{2 \omega\left|U_{0}\right|}
$$

meaning the absence of absorption effects in vacuo (for this dimension-5 theory). The latter behavior can also be inferred directly from Eq. (77), since $\Delta \omega=\omega_{+}-\omega_{-}=0$, for $\mu=\epsilon=1$, corresponding to the disappearance of frequency ranges where absorption occurs.

Furthermore, the second refractive index of Eq. (79) does not have a well-defined limit for $U_{0} \mapsto 0$. In vacuo, such modes are sometimes called spurious and their occurrence is characteristic for higher-derivative theories (see, e.g., $[71,74,75]$ for detailed investigations in the nonminimal electromagnetic sector of the SME). They can be interpreted as high-energy effects decoupling from the theory at low energies. However, a finite $U_{0}$ in macroscopic media, that is, $m\left|U_{0}\right| \sim \mathcal{O}(1)$ (with the electron mass $m$ ), is realistic. Then, the second refractive index is not necessarily suppressed for low energies in continuous media, but must be considered on an equal footing with the remaining modes. This behavior will become more transparent for the purely spacelike case to be investigated below.

\section{Propagation modes}

In order to examine the propagation modes for the purely timelike sector, we can employ Eq. (75b) in the matrix of Eq. (68), yielding

$$
\begin{aligned}
{\left[M_{i j}\right]=} & -\left(\begin{array}{ccc}
n_{1}^{2} & n_{1} n_{2} & n_{1} n_{3} \\
n_{1} n_{2} & n_{2}^{2} & n_{2} n_{3} \\
n_{1} n_{3} & n_{2} n_{3} & n_{3}^{2}
\end{array}\right) \\
& +2 \mu \omega U_{0}\left(n^{2}-1\right)\left(\begin{array}{ccc} 
\pm n & \mathrm{i} n_{3} & -\mathrm{i} n_{2} \\
-\mathrm{i} n_{3} & \pm n & \mathrm{i} n_{1} \\
\mathrm{i} n_{2} & -\mathrm{i} n_{1} & \pm n
\end{array}\right) .
\end{aligned}
$$

Solving $M_{i j} E^{j}=0$, one finds

$$
\begin{aligned}
& E_{y}=\frac{ \pm \mathrm{i} n_{3} n-n_{1} n_{2}}{n^{2}-n_{1}^{2}} E_{x}, \\
& E_{z}=\frac{\mp i n_{2} n-n_{1} n_{3}}{n^{2}-n_{1}^{2}} E_{x},
\end{aligned}
$$

such that the normalized electric fields $\mathbf{E}_{ \pm}$of the propagating waves are given by

$$
\mathbf{E}_{ \pm}=\frac{1}{\sqrt{2} n \sqrt{n^{2}-n_{1}^{2}}}\left(\begin{array}{c}
n^{2}-n_{1}^{2} \\
\pm \mathrm{i} n_{3} n-n_{1} n_{2} \\
\mp \mathrm{i} n_{2} n-n_{1} n_{3}
\end{array}\right) \text {. }
$$

The latter coincide exactly with those of Eq. (30) except of the labels being switched. Basically, for the timelike configuration, the electric-field modes of the MCFJ and MCFJ-type higher-derivative electrodynamics are the same, despite the different refractive indices of these theories. Note that $\mathbf{E}_{ \pm}$of Eqs. (30), (82) do not depend on $V_{0}$ and $U_{0}$, respectively. The refractive index illustrated in Fig. 4 is associated with the electric field $\mathbf{E}_{-}$of Eq. (82), whereas those of Figs. 5, 6 are linked to $\mathbf{E}_{+}$.

For a direct physical interpretation of these propagating modes, let us choose again a convenient coordinate system where propagation occurs along the $z$ axis, i.e., let $\mathbf{n}$ be given by Eq. (31). In this system, the normalized electric fields are

$$
\mathbf{E}_{ \pm}=\frac{1}{\sqrt{2}}\left(\begin{array}{c}
1 \\
\pm \mathrm{i} \\
0
\end{array}\right)
$$

which are the same as those stated in Eq. (32). These are polarization vectors for a left-handed and right-handed circular polarization, respectively, typical of optically active media. Such an optical activity can be expressed in terms of the rotatory power of Eq. (8), if the refractive indices $n_{+}$and $n_{-}$are known. It is worthwhile to note that, although Eq. (75c) provides, in general, three refractive indices, there are only two distinct electric-field configurations, those of Eq. (83). There are still three propagating modes, one associated with each refractive index. We will come back to this aspect in the forthcoming section, too. 


\section{Purely spacelike case}

Let us now consider the purely spacelike scenario for the background vector, $U_{0}=0$ and $\mathbf{U} \neq \mathbf{0}$, and also $\tilde{\epsilon} \mapsto \epsilon$ (setting $\sigma=0$ ). Then Eq. (69) yields

$$
\begin{aligned}
0= & \epsilon\left(n^{2}-\mu \epsilon\right)^{2}-4\left(n^{2}-1\right)^{2} \mu \omega^{2} \\
& \times\left[\left(\mu \epsilon-n^{2}\right) \mathbf{U}^{2}+(\mathbf{n} \cdot \mathbf{U})^{2}\right] .
\end{aligned}
$$

Implementing $\mathbf{n} \cdot \mathbf{U}=n|\mathbf{U}| \cos \theta$ in Eq. (84), we obtain

$$
n^{2}-\mu \epsilon= \pm 2 \mu \omega\left(n^{2}-1\right)|\mathbf{U}| \alpha,
$$

where we have defined

$$
\alpha^{2} \equiv 1-\frac{n^{2}}{\mu \epsilon} \sin ^{2} \theta
$$

With this parametrization, we can straightforwardly analyze two special cases: (i) the perpendicular configuration where $\mathbf{n} \cdot \mathbf{U}=0$ and $\sin ^{2} \theta=1$; (ii) the longitudinal configuration with $\sin ^{2} \theta=0$ and $\mathbf{n} \cdot \mathbf{U}= \pm|\mathbf{n}||\mathbf{U}|$ where the plus (minus) sign holds for $\mathbf{n}$ parallel (antiparallel) to $\mathbf{U}$. These choices can provide some insights on the behavior of electromagnetic-wave propagation.

To obtain the propagation modes, we again work in a coordinate system where Eq. (31) holds. Then the matrix (68) simplifies as

$$
\left[M_{i j}\right]=\left(\begin{array}{ccc}
n_{3}^{2}-\mu \epsilon & -\mathrm{i} \mu \epsilon \beta(\omega) U_{3} & \mathrm{i} \mu \epsilon \beta(\omega) U_{2} \\
\mathrm{i} \mu \epsilon \beta(\omega) U_{3} & n_{3}^{2}-\mu \epsilon & -\mathrm{i} \mu \epsilon \beta(\omega) U_{1} \\
-\mathrm{i} \mu \epsilon \beta(\omega) U_{2} & \mathrm{i} \mu \epsilon \beta(\omega) U_{1} & -\mu \epsilon
\end{array}\right),
$$

where $\beta(\omega)=2 \omega\left(n_{3}^{2}-1\right) / \epsilon$. For each case parametrized with $\alpha$, we can insert Eq. (85) into Eq. (86) and solve $M_{i j} E^{j}=0$ to achieve the electric fields of the corresponding modes.

\section{U-perpendicular configuration}

First, we consider the orthogonal configuration, i.e., $\mathbf{U} \perp \mathbf{n}$ and $\sin ^{2} \theta=1$, so that Eq. (85) becomes

$$
n^{2}-\mu \epsilon= \pm 2 \mu \omega\left(n^{2}-1\right)|\mathbf{U}| \sqrt{1-\frac{n^{2}}{\mu \epsilon}}
$$

which can be written as

$\left(n^{2}-\mu \epsilon\right)\left[4 \mu^{2} \omega^{2} \mathbf{U}^{2}\left(n^{2}-1\right)^{2}+\mu \epsilon\left(n^{2}-\mu \epsilon\right)\right]=0$,

implying $n^{2}=\mu \epsilon$ and

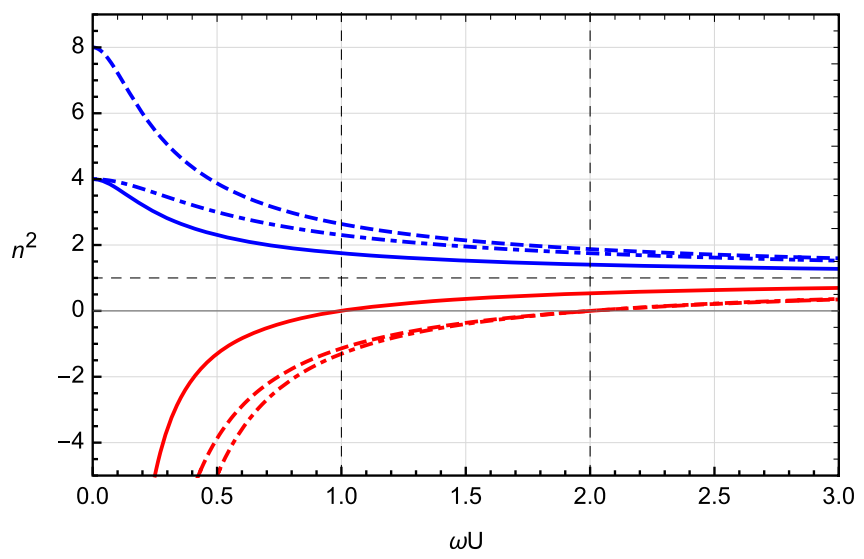

FIG. 8. Behavior of the refractive indices $n_{ \pm}^{2}$ of Eq. (90) in terms of $\omega U$ where $U=|\mathbf{U}|$. Blue curves (above the horizontal dashed line) represent $n_{+}^{2}$, while red curves (below the horizontal dashed line) depict $n_{-}^{2}$. For solid lines, $\mu=2$ and $\epsilon=2$; for dashed lines, $\mu=2$ and $\epsilon=4$; for dashed-dotted lines, $\mu=1$ and $\epsilon=4$. Gray vertical dashed lines indicate $\omega_{-} U=1$ and $\omega_{-} U=2$, respectively, where $\omega_{-}$is given by Eq. (91).

$$
\begin{aligned}
0= & 4 \mu^{2} \omega^{2} \mathbf{U}^{2} n^{4}+\left(\mu \epsilon-8 \mu^{2} \omega^{2} \mathbf{U}^{2}\right) n^{2} \\
& -\mu^{2} \epsilon^{2}+4 \mu^{2} \omega^{2} \mathbf{U}^{2} .
\end{aligned}
$$

The first solution, $n^{2}=\mu \epsilon$, corresponds to the ordinary refractive index of Maxwell electrodynamics in macroscopic media that we denote as $n_{0}=\sqrt{\mu \epsilon}$. On the other hand, Eq. (89) captures information stemming from the higher-derivative term of Eq. (58) and the background $\mathbf{U}$, leading to the following solutions:

$$
n_{ \pm}^{2}=1+f_{ \pm},
$$

where

$$
\begin{gathered}
f_{ \pm}=\frac{\epsilon}{8 \mu \omega^{2} \mathbf{U}^{2}}(-1 \pm \sqrt{1+\Upsilon}), \\
\Upsilon=16 \mu^{2} \omega^{2} \mathbf{U}^{2}\left(1-\frac{1}{\mu \epsilon}\right) .
\end{gathered}
$$

The behavior of $n_{ \pm}^{2}$ in terms of the dimensionless parameter $\omega|\mathbf{U}|$ is presented in Fig. 8. We notice that $n_{+}$ is real in the entire frequency domain and exhibits anomalous dispersion. Furthermore, the function $n_{-}^{2}$ has a simple root,

$$
\omega_{-}=\frac{\epsilon}{2|\mathbf{U}|} .
$$

The latter is interpreted as a critical value [cf. Eq. (41)], below which $n_{-}$is purely imaginary, whereupon no propagation occurs. Above $\omega_{-}$, the refractive index $n_{-}$ 
becomes real. As a consequence, electromagnetic waves can propagate in this regime.

The first vertical dashed line, located at the value $\omega_{-}|\mathbf{U}|=1$, separates the absorption and propagation zones for the mode represented by the solid red line. The second vertical dashed line, in $\omega_{-}|\mathbf{U}|=2$, does so for the modes depicted by the dashed and dashed-dotted lines. In detail, we observe that:

(i) For $0<\omega<\omega_{-}$: the refractive index $n_{+}$is real and $n_{-}$is purely imaginary; thus, only the mode associated with $n_{+}$propagates in this range.

(ii) For $\omega>\omega_{-}$: one has $n_{ \pm}^{2}>0$ and both modes propagate.

(iii) In the limit of very low frequencies, $\omega|\mathbf{U}| \mapsto 0$, it holds that $n_{+}=\sqrt{\mu \epsilon}$, recovering the usual refractive index of a simple continuous medium in standard electrodynamics.

(iv) In the limit of very high frequencies, $\omega|\mathbf{U}| \mapsto \infty$, the behavior of the refractive indices is

$$
n_{ \pm}=1 \pm \frac{1}{4 \omega|\mathbf{U}|} \sqrt{\epsilon\left(\epsilon-\frac{1}{\mu}\right)} .
$$

Unsurprisingly, the high-frequency behavior of Eq. (92), $n_{ \pm} \mapsto 1$, differs from that of the refractive indices of MCFJ theory of Eq. (40) in macroscopic media, which is given by $n_{ \pm} \mapsto \sqrt{\mu \epsilon}$. Thus, the impact of a nontrivial permeability and permittivity is suppressed in this regime of the MCFJ-type theory in Eq. (60) endowed with higher-derivative operators.

With regards to the propagating modes, Eq. (85b) yields

$$
\alpha_{ \pm}=\sqrt{1-\frac{n_{ \pm}^{2}}{\mu \epsilon}}=\sqrt{1-\frac{1+f_{ \pm}}{\mu \epsilon}},
$$

indicating different values of $\alpha$ for the distinct refractive indices $n_{ \pm}$of Eq. (90). Taking $\mathbf{n}$ as given in Eq. (31), the background has the form $\mathbf{U}=\left(U_{1}, U_{2}, 0\right)$ such that $\mathbf{n} \cdot \mathbf{U}=0$. The following propagation modes are then achieved:

$$
\begin{aligned}
\mathbf{E}_{ \pm} & =E_{0}^{\prime}\left(\begin{array}{c}
U_{2} \\
-U_{1} \\
-2 \mathrm{i} \omega f_{ \pm}\left(U_{1}^{2}+U_{2}^{2}\right) / \epsilon
\end{array}\right) \\
& =\tilde{E}_{0}^{\prime}\left(\hat{\mathbf{U}} \times \hat{\mathbf{n}}-2 \mathrm{i} \omega f_{ \pm} \frac{|\mathbf{U}|}{\epsilon} \hat{\mathbf{n}}\right),
\end{aligned}
$$

with $f_{ \pm}$given by Eq. (90b) and the unit vector $\hat{\mathbf{U}}$ pointing along the direction of $\mathbf{U}$. In case we choose the background vector of the simple form $\mathbf{U}=\left(0, U_{2}, 0\right)$, Eq. (94) provides

$$
\mathbf{E}_{ \pm}=E_{0}^{\prime}\left(\begin{array}{c}
1 \\
0 \\
-2 \mathrm{i} \omega f_{ \pm} U_{2} / \epsilon
\end{array}\right)
$$

The latter correspond to transverse, linear polarization modes with additional longitudinal components, in analogy to the mode $\mathbf{E}_{-}$of Eq. (44). Now, by comparing Eq. (94) to Eq. (43) obtained for the MCFJ theory in macroscopic matter, we spot intriguing similarities. Our interpretation is that the single mode of Eq. (43) splits into the two of Eq. (94) as a result of the higher-derivative nature of this theory. To understand these modes better, it is reasonable to perform Taylor expansions for $\mathbf{U} \mapsto \mathbf{0}$. Investigating the behavior of $f_{ \pm}$in Eq. (90b) provides

$$
f_{+} \simeq \epsilon \mu-1, \quad f_{-} \simeq-\frac{\epsilon}{4 \mu \omega^{2} \mathbf{U}^{2}}+1-\epsilon \mu,
$$

giving rise to

$$
n_{+} \mapsto \sqrt{\mu \epsilon}, \quad n_{-} \mapsto \sqrt{2-\frac{\epsilon}{4 \mu \omega^{2} \mathbf{U}^{2}}-\epsilon \mu}
$$

As a consequence, the mode described by $\mathbf{E}_{+}$has a well-defined limit for $\mathbf{U} \mapsto 0$, whereas the second mode associated with $\mathbf{E}_{-}$does not. Here it is also evident that $n_{-}$ becomes complex in this regime. So such as for the purely timelike sector, we again encounter a mode whose counterpart in vасио would frequently be denoted as spurious. The situation is different in macroscopic matter, though, because $m|\mathbf{U}| \sim \mathcal{O}(1)$ can be realistic. As before, the second mode must be interpreted as a regular, propagating mode.

Finally, we discuss the first solution $n_{0}=\sqrt{\mu \epsilon}$ of Eq. (88). In this case, Eq. (85b) provides $\alpha=0$. Hence, $M_{i j} E^{j}=0$ implies

$$
\left(\begin{array}{ccc}
0 & 0 & \mathrm{i} U_{2} \\
0 & 0 & -\mathrm{i} U_{1} \\
-\mathrm{i} U_{2} & \mathrm{i} U_{1} & -1 / \beta
\end{array}\right)\left(\begin{array}{c}
E_{x} \\
E_{y} \\
E_{z}
\end{array}\right)=0,
$$

yielding the following propagating mode:

$$
\mathbf{E}_{0}=\frac{1}{|\mathbf{U}|}\left(\begin{array}{c}
U_{1} \\
U_{2} \\
0
\end{array}\right)=\hat{\mathbf{U}}
$$

The latter is a linearly polarized mode related to the refractive index $n_{0}=\sqrt{\mu \epsilon}$ and it is perpendicular to the propagation direction of Eq. (31). Also, one finds $\mathbf{E}_{0} \cdot \mathbf{E}_{ \pm}^{*}=0$, with $\mathbf{E}_{ \pm}$given by Eq. (94). The mode of Eq. (99) is equivalent to that of Eq. (42) found for MCFJ theory in macroscopic matter. Thus, this particular mode remains unaffected by the presence of 
the additional derivatives in the CFJ-type field operator of Eq. (58). Also, even in the limit $\mathbf{U} \mapsto 0$, the electric fields $\mathbf{E}_{0}, \mathbf{E}_{ \pm}$are still governed by the direction $\hat{\mathbf{U}}$. However, $\hat{\mathbf{U}}$ does then not indicate a preferred direction, anymore. Instead, the components $U_{1}, U_{2}$ take the role of parametrizing the plane orthogonal to the propagation direction $\hat{\mathbf{n}}$.

In total, the number of the physical modes in the MCFJtype theory defined by Eq. (60) amounts to 3. Two of these approach the behavior of a standard isotropic medium in the limit $\mathbf{U} \mapsto \mathbf{0}$. In particular, it is the mode associated with $n_{+}$in Eq. (97) and that linked to $n_{0}=\sqrt{\mu \epsilon}$ of Eq. (88). Having three propagating modes does not indicate a breakdown of gauge invariance of the theory defined by Eq. (60). The operator of Eq. (58) is clearly gaugeinvariant. The third mode originates from the presence of the d'Alembertian in Eq. (58) increasing the polynomial order of the dispersion equation. In vacuo, the third mode could be denoted as spurious, but this technical term is misleading in macroscopic matter where the coefficients $m \mathbf{U}$ can take values of $\mathcal{O}(1)$.

As the associated modes are not circularly polarized, birefringence for this case is better characterized in terms of the phase shift per unit length given by Eq. (45) instead of the rotatory power in Eq. (8). We introduce

$$
\frac{\Delta_{a, b}}{d} \equiv \frac{2 \pi}{\lambda_{0}}\left(n_{a}-n_{b}\right),
$$

where $a, b \in\{0,+,-\}$. Since there are three propagating modes for $\omega>\omega_{-}$, we can define the following phase shifts acquired after propagation (divided by the propagation distance $d$ ):

$$
\begin{aligned}
& \frac{\Delta_{ \pm, 0}}{d}=\frac{2 \pi}{\lambda_{0}}\left[\sqrt{1+f_{ \pm}}-\sqrt{\mu \epsilon}\right], \\
& \frac{\Delta_{+,-}}{d}=\frac{2 \pi}{\lambda_{0}}\left[\sqrt{1+f_{+}}-\sqrt{1+f_{-}}\right],
\end{aligned}
$$

which are valid in the range where $n_{-}$is real, i.e., $\omega|\mathbf{U}|>\epsilon / 2$. In the limit of high frequencies, $(\omega|\mathbf{U}|)^{-1} \ll$ 1, Eq. (101) yields

$$
\begin{aligned}
& \frac{\Delta_{ \pm, 0}}{d}=\frac{2 \pi}{\lambda_{0}}(1-\sqrt{\mu \epsilon}) \pm \frac{\Delta}{2 d}, \\
& \frac{\Delta_{+,-}}{d}=\frac{\Delta}{d},
\end{aligned}
$$

with

$$
\frac{\Delta}{d} \equiv \frac{\pi}{\lambda_{0} \omega|\mathbf{U}|} \sqrt{\epsilon\left(\epsilon-\frac{1}{\mu}\right)} .
$$

Comparing the modes labeled with \pm to the standard mode, there is a zeroth-order contribution that only involves the permittivity and permeability of the medium.

For $\omega<\omega_{-}($or $\omega|\mathbf{U}|<\epsilon / 2), n_{-}$is purely imaginary. Then from Eq. (90), $n_{-}$is rewritten as

$$
n_{-}=\mathrm{i} \sqrt{-1-f_{-}} .
$$

Since $\operatorname{Im}\left(n_{+}\right)=0$ for the full frequency domain, only the mode labeled with the minus sign undergoes attenuation, which is quantified by the absorption coefficient, $\gamma=2 \omega \operatorname{Im}\left(n_{-}\right)$, that is

$$
\gamma=2 \omega \sqrt{\frac{\epsilon}{8 \mu \omega^{2} \mathbf{U}^{2}}(1+\sqrt{1+\Upsilon})-1},
$$

with $\Upsilon$ given by Eq. (90c). In the limit of low frequencies, $\omega|\mathbf{U}| \ll 1$, Eq. (104) can be expanded as

$$
\gamma \simeq \frac{1}{|\mathbf{U}|} \sqrt{\frac{\epsilon}{\mu}}\left[1+2\left(1-\frac{2}{\mu \epsilon}\right) \omega^{2} \mu^{2}|\mathbf{U}|^{2}\right] .
$$

It is important to note that the absorption coefficient of Eq. (105) is evaluated in the limit $\omega|\mathbf{U}| \ll 1$, while the phase shift in Eq. (102) is determined in the opposite limit $(\omega|\mathbf{U}|)^{-1} \ll 1$. Attenuation takes place for a purely imaginary $n_{-}$and birefringence occurs when $n_{-}$is real. The condition $\omega|\mathbf{U}|=\epsilon / 2$ states a clear cutoff separating the frequency regimes for each effect from each other.

\section{U-longitudinal configuration}

Let us now consider the configurations where $\sin \theta=0$, i.e., $\mathbf{n}$ and $\mathbf{U}$ are parallel or antiparallel, for which Eq. (84) is equivalent to

$$
\begin{aligned}
0= & \left(1-4 \mu^{2} \omega^{2} \mathbf{U}^{2}\right) n^{4}-2\left(\mu \epsilon-4 \mu^{2} \omega^{2} \mathbf{U}^{2}\right) n^{2} \\
& +\mu^{2} \epsilon^{2}-4 \mu^{2} \omega^{2} \mathbf{U}^{2},
\end{aligned}
$$

whose solutions for $n^{2}$ are

$$
n_{ \pm}^{2}=\frac{\mu(\epsilon \pm 2 \omega|\mathbf{U}|)}{1 \pm 2 \mu \omega|\mathbf{U}|}
$$

The behavior of $n_{ \pm}^{2}$ in terms of the dimensionless parameter $\omega|\mathbf{U}|$ is displayed in Fig. 9, for some parameter values. In this scenario, the mode associated with $n_{+}$exhibits anomalous dispersion and propagates in the full frequency range, since $n_{+}^{2}>0$.

The mode associated with $n_{-}^{2}$ has two branches. In the superior branch, defined in the frequency range $0<\omega<\omega_{0}$, the mode propagates, with $n_{-}^{2}$ increasing very rapidly with $\omega$. Here, 


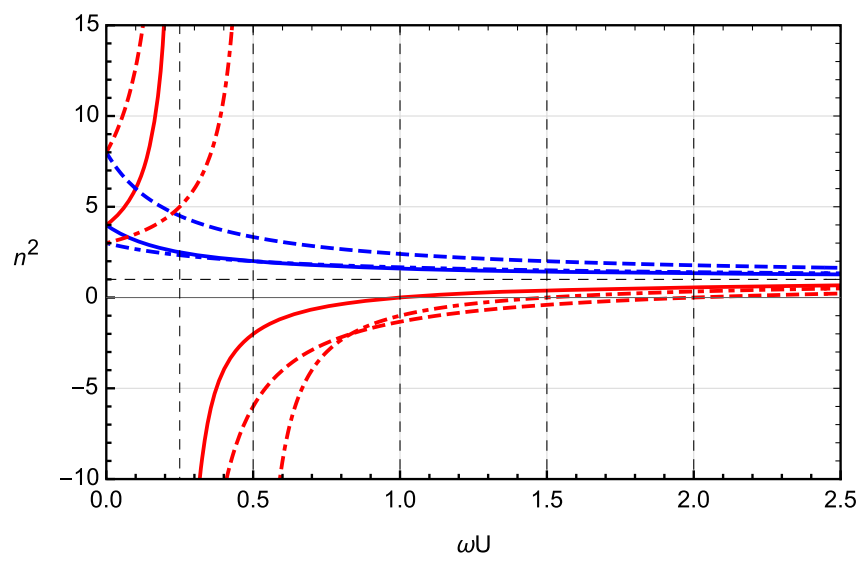

FIG. 9. Plot of $n_{+}^{2}$ of Eq. (107) in terms of $\omega U$ with $U=|\mathbf{U}|$. The blue curves, which are positive and monotonically decreasing for the entire frequency range, represent $n_{+}^{2}$. The red lines, constituted by positive upper and negative lower branches, illustrate $n_{-}^{2}$. Solid lines: $\mu=2$ and $\epsilon=2$; dashed lines: $\mu=2$ and $\epsilon=4$; dashed-dotted lines; $\mu=1$ and $\epsilon=3$. Gray vertical dashed lines indicate $\omega U \in\{1 / 4,1 / 2,1,3 / 2,2\}$.

$$
\omega_{0}=\frac{1}{2 \mu|\mathbf{U}|},
$$

is the value for which $n_{-}^{2}$ diverges. In Fig. 9, the first vertical dashed line, given by $\omega_{0}|\mathbf{U}|=1 / 4$, is asymptotic to the red solid as well as the red dashed curve where the associated functions have singularities at this point and change their signs. The second vertical dashed line is in $\omega_{0}|\mathbf{U}|=1 / 2$, being asymptotic to both the red upper and lower dashed-dotted curves. When $\omega>\omega_{0}$ one has $n_{-}^{2}<0$ whose lower branch becomes a purely imaginary refractive index $n_{-}$, representing a nonpropagating mode. This behavior is characteristic in the range $\omega_{0}<\omega<\omega_{-}$, with

$$
\omega_{-}=\frac{\epsilon}{2|\mathbf{U}|},
$$

being the root of Eq. (107). Equation (109) stands for the cutoff frequency above which the mode associated with $n_{-}$ propagates. The third and fourth vertical dashed lines, given by $\omega_{-}|\mathbf{U}|=1$ and $\omega_{-}|\mathbf{U}|=1.5$, indicate the beginning of the propagation regime for the red solid curve and the red dashed curve, respectively.

Regarding the U-longitudinal propagation modes, for which $\mathbf{n} \cdot \mathbf{U}= \pm n|\mathbf{U}|$, one takes $\alpha=1$ as well as $\mathbf{U}=$ $\left(0,0, U_{3}\right)$ for $\mathbf{n}$ given by Eq. (31). In this case, the resulting modes are

$$
\mathbf{E}_{ \pm}=\frac{1}{\sqrt{2}}\left(\begin{array}{c}
1 \\
\mp \mathrm{i} \\
0
\end{array}\right)
$$

representing right-handed and left-handed circularly polarized waves, respectively. Hence, when $\mathbf{U}$ and $\mathbf{n}$ point along the same direction, the modes become transverse again such that their polarizations are perpendicular to $\mathbf{n}$.

Now, in order to describe birefringence effects, we evaluate the rotatory power by inserting Eq. (107) into Eq. (8), that is,

$\delta=-\frac{\sqrt{\mu \epsilon}}{2} \omega\left[\frac{g_{+}}{\sqrt{1+2 \mu \omega|\mathbf{U}|}}-\frac{g_{-}}{\sqrt{1-2 \mu \omega|\mathbf{U}|}}\right]$

where

$$
g_{ \pm}=\sqrt{1 \pm \frac{2 \omega}{\epsilon}|\mathbf{U}|} .
$$

The latter result holds for the regions where $n_{-}$is real, that is, for $\omega<\omega_{0}$ and $\omega>\omega_{-}$, according to Fig. 9. In the limit $\omega|\mathbf{U}| \ll 1$, Eq. (111) provides a rotatory power nonlinear in the frequency, namely:

$$
\delta \simeq \sqrt{\frac{\mu}{\epsilon}}(\mu \epsilon-1) \omega^{2}|\mathbf{U}| .
$$

As already mentioned, the refractive index $n_{-}$is purely imaginary in the range $\omega_{0}<\omega<\omega_{-}$, constituting an absorption zone, which is explicitly given by

$$
\frac{1}{2 \mu|\mathbf{U}|}<\omega<\frac{\epsilon}{2|\mathbf{U}|}
$$

In this regime the refractive index reads

$$
n_{-}=\mathrm{i} \sqrt{\mu \epsilon} \sqrt{\frac{1-2 \omega|\mathbf{U}| / \epsilon}{2 \mu \omega|\mathbf{U}|-1}},
$$

and the corresponding dichroism coefficient is

$$
\delta_{\mathrm{d}}=\frac{\sqrt{\mu \epsilon}}{2} \omega \sqrt{\frac{1-2 \omega|\mathbf{U}| / \epsilon}{2 \mu \omega|\mathbf{U}|-1}} .
$$

\section{FINAL REMARKS}

In this work, we examined an electrodynamics of continuous media based on Maxwell equations modified by $C P T$-odd terms, whereas the usual constitutive relations $\mathbf{D}=\epsilon \mathbf{E}$ and $\mathbf{H}=\mu^{-1} \mathbf{B}$ were assumed to hold. At first, we reviewed some basic properties of the MCFJ model, followed by an analysis of the dimension-five higherderivative extension of MCFJ electrodynamics. Our general focus was on describing electromagnetic-wave propagation in matter governed by these $C P T$-odd modifications.

In Sec. III, we examined MCFJ electrodynamics, given by the Lagrangian of Eq. (11), in a continuous medium with a fixed background $V^{\mu}=\left(V^{0}, \mathbf{V}\right)$ present. To analyze the 
propagation behavior of electromagnetic waves, we obtained the dispersion relations and the refractive indices for two scenarios: (i) a timelike background, $V^{\mu}=\left(V^{0}, \mathbf{0}\right)$ and (ii) a spacelike background, $V^{\mu}=(0, \mathbf{V})$. For scenario (i), the refractive indices are always real, giving rise to propagation without losses as well as birefringence. The corresponding rotatory power, $\delta=-\mu V_{0} / 2$, is frequencyindependent. For scenario (ii), one refractive index, $n_{+}$, is always real, while the other, $n_{-}$, may be complex, corresponding to an absorption regime. In this case, birefringence and dichroism occur in different frequency ranges. The rotatory power and dichroism coefficient are both frequency-dependent.

In Sec. IV, we considered an electrodynamics in a ponderable medium modified by a MCFJ-type higherderivative term of dimension five, given by the Lagrangian of Eq. (60). After writing up the altered Maxwell equations, a sixth-order dispersion equation was achieved. In the purely timelike scenario, $U^{\mu}=\left(U^{0}, \mathbf{0}\right)$, studied in Sec. IV B, we obtained a third-order equation in the refractive index $n$ providing three solutions. One solution is real for any frequency, while the remaining two are complex for some frequency range (absorption range). This behavior occurs even for a dielectric nonconducting substrate. Such an effect is represented, for example, by Figs. 5 and 6, where the graphs indicate that $\operatorname{Im}[n(\omega)] \neq 0$ in the absorption range $\omega_{-}<\omega<\omega_{+}$, with $\omega_{ \pm}$given in Eq. (77). That property is entirely ascribed to the higher-derivative coupling term, since the usual MCFJ electrodynamics in ponderable media does not exhibit an absorption regime for a purely timelike background. Comparing Figs. 1 and 7 with each other allows us to notice the differences between the propagating modes in the usual and higher-derivative timelike case. Furthermore, the propagation modes obtained correspond to left-handed and right-handed circular polarizations [see Sec. IV B 1].

In Sec. IV C, we addressed the purely spacelike scenario, governed by an involved dispersion relation. It was analyzed for two particular cases: (a) the perpendicular configuration, where $\mathbf{n} \cdot \mathbf{U}=0$, and (b) the longitudinal configurations, $\mathbf{n} \cdot \mathbf{U}= \pm n|\mathbf{U}|$. In scenario (a), one finds $n_{+}^{2}>0$ for all frequencies, which indicates the absence of absorption for this mode. On the other hand, $n_{-}$becomes purely imaginary for $\omega<\omega_{-}$, with $\omega_{-}$defined in Eq. (91). Absorption occurs in this range for the mode associated with $n_{-}$(see Fig. 8). Hence, attenuation and birefringence are expected in the regions $\omega<\omega_{-}$and $\omega>\omega_{-}$, respectively. For scenario (b), the refractive index $n_{+}$is always real, as well, while $n_{-}$exhibits two distinct branches separated by the frequency $\omega_{0}$ given in Eq. (108). The upper branch, defined for $\omega<\omega_{0}$, is characterized by a region of sharp normal dispersion. In $\omega=\omega_{0}$, the refractive index $n_{-}$diverges. In the lower branch, the mode associated with $n_{-}$turns complex and returns to the propagation regime for $\omega>\omega_{-}$. This mode possesses different physical behaviors (propagation or absorption). Birefringence occurs for $\omega<\omega_{0}$ and $\omega>\omega_{-}$, while absorption takes place for $\omega_{0}<\omega<\omega_{-}$. In both ranges, the acquired phaseshift between different modes and the absorption coefficient are frequency-dependent.

In order to compare the spacelike configurations of the dimension-three and five MCFJ electrodynamics, we examined Figs. 2 and 8. The dimension-three model shows normal dispersion, while in the dimension-five framework modes emerge that exhibit both anomalous and normal dispersion. The absorption zones are qualitatively analogous to each other in both cases. Comparing Figs. 3 and 9, we notice that dimension-three and five modes are characterized by normal and anomalous dispersion, while only the higher-derivative model exhibits two branches of normal dispersion. In the limit of high frequencies, one has $n_{ \pm}^{2} \mapsto \mu \epsilon$ based on Eq. (36) for dimension-three MCFJ electrodynamics and $n_{ \pm}^{2} \mapsto 1$ inferred from Eqs. (90), (107) for dimension-five MCFJ-type electrodynamics. These findings allow us to distinguish between the two models. Therefore, the presence of higher derivatives implies a richer plethora of frequency-dependent propagating modes.

\section{ACKNOWLEDGMENTS}

The authors thank the anonymous referee for helpful comments that contributed to improving the paper and clarifying some results. The authors also express their gratitude to FAPEMA, CNPq, and CAPES (Brazilian research agencies) for invaluable financial support. In particular, M. M.F. is supported by FAPEMA Universal Grant No. 01187/18 and CNPq Produtividade No. 311220/ 2019-3. M. S. appreciates support by FAPEMA Universal Grant No. 00830/19 and CNPq Produtividade No. 312201/ 2018-4. Furthermore, the authors are indebted to CAPES/ Finance Code 001.

\section{APPENDIX A: COVARIANT MAXWELL EQUATIONS IN MATTER}

Here we derive the Maxwell equations and the constitutive relations from Eqs. (2a) and (2b). Equation (2b) implies

$$
\begin{aligned}
G^{0 i} & =\frac{1}{2} \chi^{0 i \alpha \beta} F_{\alpha \beta}, \\
G^{0 i} & =\frac{1}{2} \chi^{0 i 0 j} F_{0 j}+\frac{1}{2} \chi^{0 i j 0} F_{j 0}+\frac{1}{2} \chi^{0 i m n} F_{m n},
\end{aligned}
$$

which can be simplified by using the symmetry properties of the tensor $\chi^{\mu \nu \varrho \sigma}$, i.e., Eq. (3b). We also implement

$$
F_{0 i}=-F_{i 0}=E^{i}, \quad F_{m n}=-\epsilon_{m n k} B^{k},
$$

where $\epsilon_{m n k}$ is the three-dimensional Levi-Civita symbol. Thus, Eq. (A1b) becomes 


$$
\begin{aligned}
G^{0 i} & =-\chi^{0 i j 0} E^{j}-\frac{1}{2} \chi^{0 i m n} \epsilon_{m n k} B^{k}, \\
G^{0 i} & =-D^{i},
\end{aligned}
$$

where we have defined the electric displacement field $D^{i}$, which involves the medium's response to applied electromagnetic fields, as

$$
D^{i}=\chi^{0 i j 0} E^{j}+\frac{1}{2} \chi^{0 i m n} \epsilon_{m n k} B^{k} .
$$

From Eq. (A3c) we can define the electric permittivity $\epsilon_{i j}$ as well as the tensor $\gamma_{i j}$ describing the magnetic contribution to the electric displacement field [see Eq. (1a)] as

$$
\epsilon_{i j} \equiv \chi^{0 i j 0}, \quad \gamma_{i k} \equiv \frac{\chi^{0 i m n} \epsilon_{m n k}}{2} .
$$

The antisymmetric nature of $\chi^{\mu \nu \alpha \beta}$ allows us to write

$$
G^{\mu \nu}=-G^{\nu \mu} .
$$

Now we can evaluate the components $G^{i j}$. In doing so, we get

$$
\begin{aligned}
& G^{i j}=\frac{1}{2} \chi^{i j \alpha \beta} F_{\alpha \beta}, \\
& G^{i j}=\frac{1}{2} \chi^{i j 0 k} F_{0 k}+\frac{1}{2} \chi^{i j k 0} F_{k 0}+\frac{1}{2} \chi^{i j m n} F_{m n},
\end{aligned}
$$

which is recast by using Eq. (3b) as well as Eq. (A2). Then,

$$
G^{i j}=-\chi^{i j k 0} E^{k}-\frac{1}{2} \chi^{i j m n} \epsilon_{m n k} B^{k} .
$$

In order to obtain a relation between $G_{i j}$ and $H^{i}$ similar to that between $F_{i j}$ and $B^{i}$, let us now contract Eq. (A7) with $\epsilon_{i j l}$ such that

$$
\epsilon_{i j l} G^{i j}=-\frac{2}{2} \epsilon_{i j l} \chi^{i j k 0} E^{k}-\frac{2}{4} \epsilon_{i j l} \chi^{i j m n} \epsilon_{m n k} B^{k},
$$

where we introduced the factor of $(2 / 2)$ in each term of Eq. (A8). The motivation for doing so will become clear shortly, as this manipulation allows us to write down an expression very similar to $F_{m n}=-\epsilon_{m n k} B^{k}$, but for the components $G_{i j}$ and $H^{i}$. Thus, we define the magnetic permeability $\mu_{i j}$ as well as $\tilde{\gamma}_{i j}$ governing the electric contribution to the magnetic field [see Eq. (1b)] as

$$
\left(\mu^{-1}\right)_{l k} \equiv \frac{1}{4} \epsilon_{i j l} \chi^{i j m n} \epsilon_{m n k}, \quad \tilde{\gamma}_{l k} \equiv \frac{\epsilon_{i j l} \chi^{i j k 0}}{2} .
$$

Then Eq. (A8) simplifies as

$$
\begin{aligned}
& \epsilon_{i j l} G^{i j}=-2\left(\mu^{-1}\right)_{l k} B^{k}-2 \tilde{\gamma}_{l k} E^{k}, \\
& \epsilon_{i j l} G^{i j}=-2 H^{l},
\end{aligned}
$$

where we have defined the magnetic field $H^{l}$, which describes the medium's response to applied electromagnetic fields via

$$
H^{l}=\left(\mu^{-1}\right)_{l k} B^{k}+\tilde{\gamma}_{l k} E^{k} .
$$

Let us contract Eq. (A10b) with $\epsilon_{l m n}$, whereupon

$$
\begin{aligned}
\epsilon_{l m n} \epsilon_{i j l} G^{i j} & =-2 \epsilon_{l m n} H^{l}, \\
G^{m n} & =-\epsilon_{m n l} H^{l},
\end{aligned}
$$

where we have used Eq. (A5).

Now that we have expressed the constitutive relations in terms of the constitutive tensor $\chi^{\mu \nu \alpha \beta}$, we can derive the field equations associated with the Lagrange density of Eq. (2a). Thus, we start by rewriting Eq. (2a):

$$
\begin{aligned}
\mathcal{L}= & -\frac{1}{8} \chi^{\mu \nu \alpha \beta} F_{\alpha \beta} F_{\mu \nu}-A_{\mu} J^{\mu}, \\
= & -\frac{1}{8} \chi^{\mu \nu \alpha \beta} \partial_{\alpha} A_{\beta} \partial_{\mu} A_{\nu}+\frac{1}{8} \chi^{\mu \nu \alpha \beta} \partial_{\alpha} A_{\beta} \partial_{\nu} A_{\mu} \\
& +\frac{1}{8} \chi^{\mu \nu \alpha \beta} \partial_{\beta} A_{\alpha} \partial_{\mu} A_{\nu}-\frac{1}{8} \chi^{\mu \nu \alpha \beta} \partial_{\beta} A_{\alpha} \partial_{\nu} A_{\mu}-A_{\mu} J^{\mu} .
\end{aligned}
$$

We rename the indices $(\nu \leftrightarrow \mu)$ in the second and fourth term of Eq. (A12) and after that we employ the symmetry property of Eq. (3a). This gives us

$\mathcal{L}=-\frac{1}{4} \chi^{\mu \nu \alpha \beta} \partial_{\alpha} A_{\beta} \partial_{\mu} A_{\nu}+\frac{1}{4} \chi^{\mu \nu \alpha \beta} \partial_{\beta} A_{\alpha} \partial_{\mu} A_{\nu}-A_{\mu} J^{\mu}$,

which can be simplified by replacing $\alpha \leftrightarrow \beta$ in the second term and using Eq. (3b). Hence, we finally obtain

$$
\mathcal{L}=-\frac{1}{2} \chi^{\mu \nu \alpha \beta} \partial_{\alpha} A_{\beta} \partial_{\mu} A_{\nu}-A_{\mu} J^{\mu} .
$$

Using the Euler-Lagrange equations

$$
\frac{\partial \mathcal{L}}{\partial A_{\kappa}}-\partial_{\rho}\left(\frac{\partial \mathcal{L}}{\partial\left(\partial_{\rho} A_{\kappa}\right)}\right)=0,
$$

one arrives at

$$
\frac{\partial \mathcal{L}}{\partial\left(\partial_{\rho} A_{\kappa}\right)}=-\frac{1}{2}\left(\chi^{\beta \alpha \rho \kappa} \partial_{\beta} A_{\alpha}+\chi^{\rho \kappa \alpha \beta} \partial_{\alpha} A_{\beta}\right),
$$

where we have relabeled $\mu \rightarrow \beta, \nu \rightarrow \alpha$ in the first term on the right-hand side. Now, we also implement Eq. (3c) in the 
first contribution, and in the second term we take advantage of Eq. (3b). Therefore, Eq. (A16) provides

$$
\frac{\partial \mathcal{L}}{\partial\left(\partial_{\rho} A_{\kappa}\right)}=-\frac{1}{2} \chi^{\rho \kappa \beta \alpha} F_{\beta \alpha}=-G^{\rho \kappa},
$$

and one also finds

$$
\frac{\partial \mathcal{L}}{\partial A_{\kappa}}=-J^{\mu} \delta_{\mu \kappa}=-J^{\kappa} .
$$

So using Eqs. (A17) and (A18) in Eq. (A15), we finally get the covariant form of the Maxwell equations in simple matter:

$$
\partial_{\rho} G^{\rho \kappa}=J^{\kappa} .
$$

Taking $\kappa=0$, one finds Gauss's law:

$$
\begin{aligned}
& \partial_{i} G^{i 0}=J^{0}, \\
& \nabla \cdot \mathbf{D}=\rho,
\end{aligned}
$$

where we have employed Eq. (A3b) and $J^{\mu}=(\rho, \mathbf{J})$. Ampère's law is obtained by taking $\kappa=i$ in Eq. (A19), that is

$$
\begin{array}{r}
\partial_{0} G^{0 i}+\partial_{j} G^{j i}=J^{i}, \\
\partial_{t}\left(-D^{i}\right)-\partial_{j}\left(\epsilon_{j i k} H^{k}\right)=J^{i},
\end{array}
$$

where we have used Eqs. (A3b), (A5), and (A11b). Applying further simplifications to Eq. (A21b), yields

$$
\begin{gathered}
\epsilon_{i j k} \partial_{j} H^{k}-\partial_{t} D^{i}=J^{i}, \\
\nabla \times \mathbf{H}-\partial_{t} \mathbf{D}=\mathbf{J} .
\end{gathered}
$$

\section{APPENDIX B: ROTATORY POWER AND DICHROISM COEFFICIENT}

As mentioned at the end of Sec. II, when the propagating modes resulting from an electromagnetic theory are lefthanded and right-handed circularly polarized waves, birefringence is characterized in terms of the rotatory power while absorption is described via the dichroism coefficient, presented in Eqs. (8) and (9), respectively. Such relations can be derived by means of the polarization vectors of a wave traveling through a medium. Consider, for instance, a linearly polarized wave propagating through a medium along the $z$ axis. Hence, the initial electric field can be written as

$$
\mathbf{E}_{i}=\mathbf{E}_{0 i} \mathrm{e}^{\mathrm{i}(k z-\omega t)},
$$

with the polarization vector (for an electric field pointing along the $x$ axis):

$$
\mathbf{E}_{0 i}=\left(\begin{array}{l}
1 \\
0 \\
0
\end{array}\right)=\frac{1}{2}\left(\begin{array}{c}
1 \\
-\mathrm{i} \\
0
\end{array}\right)+\frac{1}{2}\left(\begin{array}{l}
1 \\
\mathrm{i} \\
0
\end{array}\right),
$$

which corresponds to the sum of polarization vectors associated with left-handed and right-handed circular polarizations, respectively. After the wave passes through a distance $z$ in the medium, the final electric field is a linear combination of two components, $\mathbf{E}_{+}$and $\mathbf{E}_{-}$, with the wave vectors $\mathbf{k}_{+}$and $\mathbf{k}_{-}$, respectively. One then has

$$
\begin{aligned}
& \mathbf{E}_{f}=\mathbf{E}_{+} \mathrm{e}^{\mathrm{i}\left(k_{+} z-\omega t\right)}+\mathbf{E}_{-} \mathrm{e}^{\mathrm{i}\left(k_{-} z-\omega t\right)}, \\
& \mathbf{E}_{f}=\frac{1}{2}\left(\begin{array}{l}
1 \\
\mathrm{i} \\
0
\end{array}\right) \mathrm{e}^{\mathrm{i} k_{+} z} \mathrm{e}^{-\mathrm{i} \omega t}+\frac{1}{2}\left(\begin{array}{c}
1 \\
-\mathrm{i} \\
0
\end{array}\right) \mathrm{e}^{\mathrm{i} k \_z} \mathrm{e}^{-\mathrm{i} \omega t},
\end{aligned}
$$

which can be cast into the form

$$
\begin{aligned}
& \mathbf{E}_{f}=\frac{1}{2} \mathrm{e}^{\mathrm{i} \psi /} \mathrm{e}^{-\mathrm{i} \omega t}\left[\mathrm{e}^{-\mathrm{i} \theta}\left(\begin{array}{l}
1 \\
\mathrm{i} \\
0
\end{array}\right)+\mathrm{e}^{\mathrm{i} \theta}\left(\begin{array}{c}
1 \\
-\mathrm{i} \\
0
\end{array}\right)\right], \\
& \mathbf{E}_{f}=\mathrm{e}^{\mathrm{i} \psi} \mathrm{e}^{-\mathrm{i} \omega t}\left(\begin{array}{c}
\cos \theta \\
\sin \theta \\
0
\end{array}\right),
\end{aligned}
$$

with the quantities

$$
\begin{aligned}
\theta & \equiv-\frac{\left(k_{+}-k_{-}\right) z}{2}, \\
\psi & \equiv \frac{\left(k_{+}+k_{-}\right) z}{2} .
\end{aligned}
$$

Notice that Eq. (B3a) describes a linearly polarized wave whose polarization vector is rotated by an angle $\theta$. From Eq. (B3b), one obtains

$$
\theta=-\frac{\left(n_{+}-n_{-}\right) z \omega}{2},
$$

where we have used $\mathbf{k}=\omega \mathbf{n}$. In general, the refractive indices can be complex quantities. Because of this, one can infer from Eq. (B4)

$\frac{\theta}{z}=-\frac{\omega}{2}\left[\operatorname{Re}\left(n_{+}\right)+\mathrm{i} \operatorname{Im}\left(n_{+}\right)-\operatorname{Re}\left(n_{-}\right)-\mathrm{i} \operatorname{Im}\left(n_{-}\right)\right]$,

from which we define the specific rotatory power stated in Eq. (8) as well as the dichroism coefficient of Eq. (9). Notice that when the medium is nonbirefringent, $\theta=0$ and $\psi=k z$. Then, the form of Eq. (B1a) is recovered from Eq. (B3a). 
[1] J. D. Jackson, Classical Electrodynamics, 3rd ed. (John Wiley \& Sons, New York, 1999).

[2] A. Zangwill, Modern Electrodynamics (Cambridge University Press, New York, 2012).

[3] L. D. Landau and E. M. Lifshitz, Electrodynamics of Continuous Media, Course of Theoretical Physics, Vol. 8, 2nd ed. (Pergamon Press, New York, 1984).

[4] A. K. Bain, Crystal optics, Properties and Applications (Wiley-VCH Verlag GmbH \& Co. KGaA, Germany, 2019).

[5] G. R. Fowles, Introduction to Modern Optics, 2nd ed. (Dover Publications, INC., New York, 1975).

[6] E. Hecht, Optics, 4th ed. (Addison Wesley, San Francisco, 2002).

[7] A. A. Kurmanov, N. A. Ispulov, A. Qadir, A. Z. Zhumabekov, S. N. Sarymova, and K. R. Dossumbekov, Propagation of electromagnetic waves in stationary anisotropic media, Phys. Scr. 96, 085505 (2021).

[8] I. Yakov, Dispersion relation for electromagnetic waves in anisotropic media, Phys. Lett. A 374, 1113 (2010).

[9] K. Halterman, M. Alidoust, and A. Zyuzin, Epsilonnear-zero response and tunable perfect absorption in Weyl semimetals, Phys. Rev. B 98, 085109 (2018).

[10] R. Zu, M. Gu, L. Min, C. Hu, N. Ni, Z. Mao, J. M. Rondinelli, and V. Gopalan, Comprehensive anisotropic linear optical properties of the Weyl semimetals TaAs and NbAs, Phys. Rev. B 103, 165137 (2021).

[11] J. Krupka, Measurement of the complex permittivity, initial permeability, permeability tensor and ferromagnetic linewidth of gyromagnetic materials, Meas. Sci. Technol. 29, 092001 (2018).

[12] J. Krupka, A. Pacewicz, B. Salski, P. Kopyt, J. Bourhill, M. Goryachev, and M. Tobar, Electrodynamic improvements to the theory of magnetostatic modes in ferrimagnetic spheres and their applications to saturation magnetization measurements, J. Magn. Magn. Mater. 487, 165331 (2019).

[13] Y. T. Aladadi and M. A. S. Alkanhal, Classification and characterization of electromagnetic materials, Sci. Rep. 10, 11406 (2020).

[14] A. H. Sihvola and I. V. Lindell, Bi-isotropic constitutive relations, Microwave Opt. Technol. Lett. 4, 295 (1991).

[15] S. Ougier, I. Chenerie, A. Sihvola, and A. C. Priou, Propagation in bi-isotropic media: Effect of different formalisms on the propagation analysis, Prog. Electromagn. Res. C 09, 19 (1994), https://www.jpier.org/PIER/pier.php? paper $=9301010$.

[16] J. F. Nieves and P. B. Pal, Third electromagnetic constant of an isotropic medium, Am. J. Phys. 62, 207 (1994).

[17] P. Hillion, Manifestly covariant formalism for electromagnetism in chiral media, Phys. Rev. E 47, 1365 (1993).

[18] R. Li, J. Wang, X.-L. Qi, and S.-C. Zhang, Dynamical axion field in topological magnetic insulators, Nat. Phys. 6, 284 (2010).

[19] A. Martín-Ruiz, M. Cambiaso, and L.F. Urrutia, The magnetoelectric coupling in electrodynamics, Int. J. Mod. Phys. A 34, 1941002 (2019).

[20] A. Martín-Ruiz, M. Cambiaso, and L.F. Urrutia, Electro- and magnetostatics of topological insulators as modeled by planar, spherical, and cylindrical $\theta$ boundaries: Green's function approach, Phys. Rev. D 93, 045022 (2016).
[21] A. Lakhtakia and T. G. Mackay, Classical electromagnetic model of surface states in topological insulators, J. Nanophoton. 10, 033004 (2016).

[22] T. M. Melo, D. R. Viana, W. A. Moura-Melo, J. M. Fonseca, and A. R. Pereira, Topological cutoff frequency in a slab waveguide: Penetration length in topological insulator walls, Phys. Lett. A 380, 973 (2016).

[23] Z.-X. Li, Y. Cao, and P. Yan, Topological insulators and semimetals in classical magnetic systems, Phys. Rep. 915, 1 (2021).

[24] C. A. A. de Carvalho, Relativistic electron gas: A candidate for nature's left-handed materials, Phys. Rev. D 93, 105005 (2016); E. Reyes-Gómez, L. E. Oliveira, and C. A. A. de Carvalho, The electromagnetic response of a relativistic Fermi gas at finite temperatures: Applications to condensedmatter systems, Europhys. Lett. 114, 17009 (2016).

[25] A. Sekine and K. Nomura, Axion electrodynamics in topological materials, J. Appl. Phys. 129, 141101 (2021).

[26] M. E. Tobar, B. T. McAllister, and M. Goryachev, Modified axion electrodynamics as impressed electromagnetic sources through oscillating background polarization and magnetization, Phys. Dark Universe 26, 100339 (2019).

[27] L. H. C. Borges, A. G. Dias, A. F. Ferrari, J. R. Nascimento, and A. Yu. Petrov, Generation of axionlike couplings via quantum corrections in a Lorentz-violating background, Phys. Rev. D 89, 045005 (2014).

[28] M. E. Tobar, P. Wolf, A. Fowler, and J. G. Hartnett, New methods of testing Lorentz violation in electrodynamics, Phys. Rev. D 71, 025004 (2005); 75, 049902(E) (2007).

[29] Q. G. Bailey and V. A. Kostelecký, Lorentz-violating electrostatics and magnetostatics, Phys. Rev. D 70, 076006 (2004).

[30] F. Bopp, Eine lineare Theorie des Elektrons, Ann. Phys. (Berlin) 430, 345 (1940).

[31] B. Podolsky, A generalized electrodynamics part I-nonquantum, Phys. Rev. 62, 68 (1942).

[32] B. Podolsky and C. Kikuchi, A generalized electrodynamics part II-quantum, Phys. Rev. 65, 228 (1944).

[33] C. A. P. Galvão and B. M. Pimentel, The canonical structure of Podolsky generalized electrodynamics, Can. J. Phys. 66, 460 (1988); M. C. Bertin, B. M. Pimentel, and G. E. R. Zambrano, The canonical structure of Podolsky's generalized electrodynamics on the null-plane, J. Math. Phys. (N.Y.) 52, 102902 (2011).

[34] J. Barcelos-Neto, C. A. P. Galvão, and C. P. Natividade, Quantization of Podolsky theory in the BFV formalism, Z. Phys. C 52, 559 (1991); R. Bufalo, B. M. Pimentel, and G. E. R. Zambrano, Path integral quantization of generalized quantum electrodynamics, Phys. Rev. D 83, 045007 (2011).

[35] A. E. Zayats, Self-interaction in the Bopp-Podolsky electrodynamics: Can the observable mass of a charged particle depend on its acceleration?, Ann. Phys. (Amsterdam) 342, 11 (2014).

[36] J. Gratus, V. Perlick, and R. W. Tucker, On the self-force in Bopp-Podolsky electrodynamics, J. Phys. A 48, 435401 (2015); M. K.-H. Kiessling, Force on a point charge source of the classical electromagnetic field, Phys. Rev. D 100, 065012 (2019); 101, 109901(E) (2020). 
[37] M. Lazar, Green functions and propagation in the BoppPodolsky electrodynamics, Wave Motion 91, 102388 (2019); M. Lazar and J. Leck, Second gradient electromagnetostatics: Electric point charge, electrostatic and magnetostatic dipoles, Symmetry 12, 1104 (2020).

[38] C. A. Bonin, B. M. Pimentel, and P. H. Ortega, Multipole expansion in generalized electrodynamics, Int. J. Mod. Phys. A 34, 1950134 (2019).

[39] P. Fan, J. Xiao, and H. Qin, Gauge-symmetrization method for energy-momentum tensors in high-order electromagnetic field theories, Phys. Rev. D 104, 025013 (2021); I. G. Oliveira, J. H. Sales, and R. Thibes, Bopp-Podolsky scalar electrodynamics propagators and energy-momentum tensor in covariant and light-front coordinates, Eur. Phys. J. Plus 135, 713 (2020); P. Fan, Q. Chen, and J. Xiao, High-order field theory and weak Euler-Lagrange-Barut equation for classical relativistic particle-field systems, arXiv:2104 .04196 .

[40] J. Dai, Stability and Hamiltonian BRST-invariant deformations in Podolsky's generalized electrodynamics, Nucl. Phys. B971, 115497 (2021).

[41] R. Bufalo, B. M. Pimentel, and D. E. Soto, Causal approach for the electron-positron scattering in generalized quantum electrodynamics, Phys. Rev. D 90, 085012 (2014); G. P. de Brito, P. C. Malta, and L. P. R. Ospedal, Spin- and velocitydependent nonrelativistic potentials in modified electrodynamics, Phys. Rev. D 95, 016006 (2017).

[42] B. El-Bennich, G. Ramos-Zambrano, and E. Rojas, Podolsky propagator in the gap and bound-state equations, Phys. Rev. D 103, 076008 (2021); C.-R. Ji, A. T. Suzuki, J. H. Sales, and R. Thibes, Pauli-Villars regularization elucidated in Bopp-Podolsky's generalized electrodynamics, Eur. Phys. J. C 79, 871 (2019).

[43] D. R. Granado, A. J. G. Carvalho, A. Yu. Petrov, and P. J. Porfirio, Podolsky electrodynamics from a condensation of topological defects, Europhys. Lett. 129, 51001 (2020).

[44] T. D. Lee and G. C. Wick, Negative metric and the unitarity of the S-matrix, Nucl. Phys. B9, 209 (1969).

[45] T.D. Lee and G.C. Wick, Finite theory of quantum electrodynamics, Phys. Rev. D 2, 1033 (1970).

[46] R. Turcati and M. J. Neves, Probing features of the LeeWick quantum electrodynamics, Adv. High Energy Phys. 2014, 153953 (2014).

[47] A. Accioly, P. Gaete, J. A. Helayël-Neto, E. Scatena, and R. Turcati, Investigations in the Lee-Wick electrodynamics, Mod. Phys. Lett. A 26, 1985 (2011).

[48] R. Turcati and M. J. Neves, Complex-mass shell renormalization of the higher-derivative electrodynamics, Eur. Phys. J. C 76, 456 (2016).

[49] L. H. C. Borges, F. A. Barone, C. A. M. de Melo, and F. E. Barone, Higher order derivative operators as quantum corrections, Nucl. Phys. B944, 114634 (2019).

[50] V. A. Kostelecký and S. Samuel, Spontaneous breaking of Lorentz symmetry in string theory, Phys. Rev. D 39, 683 (1989); V. A. Kostelecký and R. Potting, CPT and strings, Nucl. Phys. B359, 545 (1991); CPT, strings, and meson factories, Phys. Rev. D 51, 3923 (1995).

[51] D. Colladay and V. A. Kostelecký, $C P T$ violation and the standard model, Phys. Rev. D 55, 6760 (1997); Lorentzviolating extension of the standard model, Phys. Rev. D 58,
116002 (1998); S. Coleman and S. L. Glashow, Highenergy tests of Lorentz invariance, Phys. Rev. D 59, 116008 (1999).

[52] V. A. Kostelecký and M. Mewes, Cosmological Constraints on Lorentz Violation in Electrodynamics, Phys. Rev. Lett. 87, 251304 (2001); Signals for Lorentz violation in electrodynamics, Phys. Rev. D 66, 056005 (2002); Sensitive Polarimetric Search for Relativity Violations in GammaRay Bursts, Phys. Rev. Lett. 97, 140401 (2006).

[53] C. A. Escobar and M. A. G. Garcia, Full CPT-even photon sector of the standard model extension at finite temperature, Phys. Rev. D 92, 025034 (2015); A. Martín-Ruiz and C. A. Escobar, Casimir effect between ponderable media as modeled by the standard model extension, Phys. Rev. D 94, 076010 (2016).

[54] P. R. A. Souza, A. F. Santos, and Faqir C. Khanna, Effects of the $C P T$-even and Lorentz violation on the Bhabha scattering at finite temperature, Ann. Phys. (Amsterdam) 428, 168451 (2021); P. R. A. Souza, A. F. Santos, S. C. Ulhoa, and Faqir C. Khanna, On Lorentz violation in $e^{-}+e^{+} \rightarrow$ $\mu^{-}+\mu^{+}$scattering at finite temperature, Phys. Lett. B 791, 195 (2019).

[55] T. Prudêncio and H. Belich, Nonorthogonality and $\kappa$ dependence eccentricity of polarized electromagnetic waves in CPT-even Lorentz violation, Adv. High Energy Phys. 2017, 3050724 (2017); K. Bakke and H. Belich, Relativistic geometric quantum phases from the Lorentz symmetry violation effects in the $C P T$-even gauge sector of Standard Model Extension, Int. J. Mod. Phys. A 30, 1550197 (2015); R. L. L. Vitória, H. Belich, and K. Bakke, Coulomb-type interaction under Lorentz symmetry breaking effects, Adv. High Energy Phys. 2017, 6893084 (2017).

[56] S. M. Carroll, G. B. Field, and R. Jackiw, Limits on a Lorentz- and parity-violating modification of electrodynamics, Phys. Rev. D 41, 1231 (1990); A. A. Andrianov and R. Soldati, Lorentz symmetry breaking in Abelian vector-field models with Wess-Zumino interaction, Phys. Rev. D 51, 5961 (1995); A. A. Andrianov and R. Soldati, Patterns of Lorentz symmetry breaking in QED by $C P T$-odd interaction, Phys. Lett. B 435, 449 (1998); A. A. Andrianov, R. Soldati, and L. Sorbo, Dynamical Lorentz symmetry breaking from a $(3+1)$-dimensional axion-Wess-Zumino model, Phys. Rev. D 59, 025002 (1998).

[57] J. Alfaro, A. A. Andrianov, M. Cambiaso, P. Giacconi, and R. Soldati, Bare and induced Lorentz and CPT invariance violations in QED, Int. J. Mod. Phys. A 25, 3271 (2010); A. A. Andrianov, D. Espriu, P. Giacconi, and R. Soldati, Anomalous positron excess from Lorentz-violating QED, J. High Energy Phys. 09 (2009) 057.

[58] A. P. Baêta Scarpelli, H. Belich, J. L. Boldo, and J. A. Helayël-Neto, Aspects of causality and unitarity and comments on vortexlike configurations in an Abelian model with a Lorentz-breaking term, Phys. Rev. D 67, 085021 (2003); L. Bonetti, L. R. dos Santos Filho, J. A. HelayëlNeto, and A. D. A. M. Spallicci, Photon sector analysis of Super and Lorentz symmetry breaking: Effective photon mass, bi-refringence and dissipation, Eur. Phys. J. C 78, 811 (2018).

[59] M. M. Ferreira, Jr., J. A. Helayël-Neto, C. M. Reyes, M. Schreck, and P.D.S. Silva, Unitarity in Stückelberg 
electrodynamics modified by a Carroll-Field-Jackiw term, Phys. Lett. B 804, 135379 (2020); L. C. T. Brito, J. C. C. Felipe, A. Yu. Petrov, and A. P. Baêta Scarpelli, No radiative corrections to the Carroll-Field-Jackiw term beyond oneloop order, Int. J. Mod. Phys. A 36, 2150033 (2021).

[60] J.-M. Chung, Lorentz- and $C P T$-violating Chern-Simons term in the functional integral formalism, Phys. Rev. D 60 , 127901 (1999); Radiatively-induced Lorentz and CPT violating Chern-Simons term in QED, Phys. Lett. B 461, 138 (1999); M. Pérez-Victoria, Exact Calculation of the Radiatively Induced Lorentz and $C P T$ Violation in QED, Phys. Rev. Lett. 83, 2518 (1999); G. Bonneau, Regularisation: Many recipes, but a unique principle: Ward identities and normalisation conditions. The case of $C P T$ violation in QED, Nucl. Phys. B593, 398 (2001); M. Pérez-Victoria, Physical (ir)relevance of ambiguities to Lorentz and CPT violation in QED, J. High Energy Phys. 04 (2001) 032; O. A. Battistel and G. Dallabona, Role of ambiguities and gauge invariance in the calculation of the radiatively induced Chern-Simons shift in extended QED, Nucl. Phys. B610, 316 (2001); O. A. Battistel and G. Dallabona, Ambiguities versus gauge invariance in the calculation of the radiatively induced Chern-Simons shift in extended QED, J. Phys. G 27, L53 (2001); O. A. Battistel and G. Dallabona, Consistency in perturbative calculations and radiatively induced Lorentz and $C P T$ violations, J. Phys. G 28, L23 (2002); J.-M. Chung and B. K. Chung, Induced Lorentz- and $C P T$-violating Chern-Simons term in QED: Fock-Schwinger proper time method, Phys. Rev. D 63, 105015 (2001); A. P. Baêta Scarpelli, M. Sampaio, M. C. Nemes, and B. Hiller, Chiral anomaly and $C P T$ invariance in an implicit momentum space regularization framework, Phys. Rev. D 64, 046013 (2001).

[61] C. Adam and F. R. Klinkhamer, Causality and CPT violation from an Abelian Chern-Simons-like term, Nucl. Phys. B607, 247 (2001).

[62] C. Adam and F. R. Klinkhamer, Photon decay in a $C P T$ violating extension of quantum electrodynamics, Nucl. Phys. B657, 214 (2003); B. Altschul, Gauge invariance and the Pauli-Villars regulator in Lorentz- and $C P T$ violating electrodynamics, Phys. Rev. D 70, 101701(R) (2004); C. Kaufhold and F. R. Klinkhamer, Vacuum Cherenkov radiation and photon triple-splitting in a Lorentz-noninvariant extension of quantum electrodynamics, Nucl. Phys. B734, 1 (2006); C. Kaufhold and F. R. Klinkhamer, Vacuum Cherenkov radiation in spacelike Maxwell-Chern-Simons theory, Phys. Rev. D 76, 025024 (2007); B. Altschul, Cerenkov radiation in a Lorentzviolating and birefringent vacuum, Phys. Rev. D 75, 105003 (2007); Absence of long-wavelength Cerenkov radiation with isotropic Lorentz and $C P T$ violation, Phys. Rev. D 90, 021701(R) (2014); K. Schober and B. Altschul, No vacuum Cerenkov radiation losses in the timelike Lorentz-violating Chern-Simons theory, Phys. Rev. D 92, 125016 (2015); R. DeCosta and B. Altschul, Mode analysis for energetics of a moving charge in Lorentz- and CPT-violating electrodynamics, Phys. Rev. D 97, 055029 (2018).

[63] Z. Qiu, G. Cao, and X.-G. Huang, Electrodynamics of chiral matter, Phys. Rev. D 95, 036002 (2017).
[64] K. Fukushima, D. E. Kharzeev, and H. J. Warringa, Chiral magnetic effect, Phys. Rev. D 78, 074033 (2008).

[65] D. E. Kharzeev, The chiral magnetic effect and anomalyinduced transport, Prog. Part. Nucl. Phys. 75, 133 (2014).

[66] D. E. Kharzeev, J. Liao, S. A. Voloshin, and G. Wang, Chiral magnetic and vortical effects in high-energy nuclear collisions-A status report, Prog. Part. Nucl. Phys. 88, 1 (2016).

[67] D. Kharzeev, K. Landsteiner, A. Schmitt, and H. U. Yee, Strongly Interacting Matter in Magnetic Fields, Lect. Notes Phys. Vol. 871 (Springer-Verlag, Berlin, Heidelberg, 2013).

[68] A. A. Zyuzin and A. A. Burkov, Topological response in Weyl semimetals and the chiral anomaly, Phys. Rev. B 86, 115133 (2012).

[69] V. A. Kostelecký and M. Mewes, Electrodynamics with Lorentz-violating operators of arbitrary dimension, Phys. Rev. D 80, 015020 (2009); M. Mewes, Higher-order Lorentz violation, in Proceedings of the 6th Meeting on CPT and Lorentz. Symmetry, edited by V. A. Kostelecký (World Scientific, Singapore, 2014); Y. Ding and V. A. Kostelecký, Lorentz-violating spinor electrodynamics and Penning traps, Phys. Rev. D 94, 056008 (2016); V. A. Kostelecký and Z. Li, Gauge field theories with Lorentzviolating operators of arbitrary dimension, Phys. Rev. D 99, 056016 (2019).

[70] M. Mewes, Optical-cavity tests of higher-order Lorentz violation, Phys. Rev. D 85, 116012 (2012).

[71] M. Schreck, Quantum field theoretic properties of Lorentzviolating operators of nonrenormalizable dimension in the photon sector, Phys. Rev. D 89, 105019 (2014).

[72] R. C. Myers and M. Pospelov, Ultraviolet Modifications of Dispersion Relations in Effective Field Theory, Phys. Rev. Lett. 90, 211601 (2003).

[73] C. M. Reyes, Unitarity in higher-order Lorentz-invariance violating QED, Phys. Rev. D 87, 125028 (2013).

[74] R. Casana, M. M. Ferreira, Jr., L. Lisboa-Santos, F. E. P. dos Santos, and M. Schreck, Maxwell electrodynamics modified by $C P T$-even and Lorentz-violating dimension-6 higherderivative terms, Phys. Rev. D 97, 115043 (2018).

[75] M. M. Ferreira, Jr., L. Lisboa-Santos, R. V. Maluf, and M. Schreck, Maxwell electrodynamics modified by a CPT-odd dimension-five higher-derivative term, Phys. Rev. D 100, 055036 (2019).

[76] T. A. M. Sampaio and E. Passos, Consistency analysis of a $C P T$-even and $C P T$-odd Lorentz-violating effective field theory in the electrodynamics at Planck scale by an influence of a background isotropic field, Int. J. Mod. Phys. A 34, 1950192 (2019).

[77] L. H. C. Borges, A. F. Ferrari, and F. A. Barone, New effects in the interaction between electromagnetic sources mediated by nonminimal Lorentz violating interactions, Eur. Phys. J. C 76, 599 (2016).

[78] A. A. Araújo. Filho and R. V. Maluf, Thermodynamic properties in higher-derivative electrodynamics, Braz. J. Phys. 51, 820 (2021); A. A. Araújo Filho and J. A. A. S. Reis, Thermal aspects of interacting quantum gases in Lorentz-violating scenarios, Eur. Phys. J. Plus 136, 310 (2021); A. A. Araújo Filho, Lorentz-violating scenarios in a thermal reservoir, Eur. Phys. J. Plus 136, 417 (2021).

[79] E. Passos, E. M. C. Abreu, M. A. Anacleto, F. A. Brito, C. Wotzasek, and C.A.D. Zarro, Lifshitz scaling to 
Lorentz-violating high derivative operator and gamma-ray bursts, Phys. Rev. D 93, 085022 (2016).

[80] A. F. Ferrari, J. R. Nascimento, and A. Yu. Petrov, Radiative corrections and Lorentz violation, Eur. Phys. J. C 80, 459 (2020); T. Mariz, J. R. Nascimento, A. Yu. Petrov, and C. M. Reyes, Quantum aspects of the higher-derivative Lorentzbreaking extension of QED, Phys. Rev. D 99, 096012 (2019); L. H. C. Borges, A. G. Dias, A. F. Ferrari, J. R. Nascimento, and A. Yu. Petrov, Generation of higher derivatives operators and electromagnetic wave propagation in a Lorentz-violation scenario, Phys. Lett. B 756, 332 (2016).

[81] J. Shibata, A. Takeuchi, H. Kohno, and G. Tatara, Theory of electromagnetic wave propagation in ferromagnetic Rashba conductor, J. App. Phys. 123, 063902 (2018).

[82] R. A. Shelby, D. R. Smith, and S. Schultz, Experimental verification of a negative index of refraction, Science 292, 77 (2001).

[83] P. M. Valanju, R. M. Walser, and A. P. Valanju, Wave Refraction in Negative-Index Media: Always Positive and Very Inhomogeneous, Phys. Rev. Lett. 88, 187401 (2002).

[84] R. S. Kshetrimayum, A brief intro to metamaterials, IEEE Potentials 23, 44 (2004).

[85] A. G. Grushin, Consequences of a condensed matter realization of Lorentz-violating QED in Weyl semi-metals, Phys. Rev. D 86, 045001 (2012).
[86] P. D. S. Silva, M. M. Ferreira, Jr., M. Schreck, and L. F. Urrutia, Magnetic-conductivity effects on electromagnetic propagation in dispersive matter, Phys. Rev. D 102, 076001 (2020).

[87] S. Kaushik, D. E. Kharzeev, and E. J. Philip, Transverse chiral magnetic photocurrent induced by linearly polarized light in mirror-symmetric Weyl semimetals, Phys. Rev. Research 2, 042011(R) (2020).

[88] E. J. Post, Formal Structure of Electromagnetics: General Covariance and Electromagnetics (Dover Publications Inc., Mineola, New York, 1997); P. Penfield, Jr. and H. A. Haus, Electrodynamics of Moving Media, MIT Press Research Monographs Vol. 40 (The MIT Press, Cambridge, MA, 1967).

[89] B. Yan and C. Felser, Topological materials: Weyl semimetals, Annu. Rev. Condens. Matter Phys. 8, 337 (2017); H. Gao, J. W. F. Venderbos, Y. Kim, and A. M. Rappe, Topological semimetals from first principles, Annu. Rev. Mat. Res. 49, 153 (2019).

[90] J. Behrends, S. Roy, M. H. Kolodrubetz, J. H. Bardarson, and A. G. Grushin, Landau levels, Bardeen polynomials, and Fermi arcs in Weyl semimetals: Lattice-based approach to the chiral anomaly, Phys. Rev. B 99, 140201(R) (2019).

[91] V. A. Kostelecký and N. Russell, Data tables for Lorentz and CPT violation, arXiv:0801.0287; Data tables for Lorentz and $C P T$ violation, Rev. Mod. Phys. 83, 11 (2011). 